\title{
MAIN BELT ASTEROIDS WITH WISE/NEOWISE. I. PRELIMINARY ALBEDOS AND DIAMETERS
}

\author{
Joseph R. Masiero ${ }^{1}$, A. K. Mainzer ${ }^{1}$, T. Grav ${ }^{2}$, J. M. Bauer ${ }^{1,3}$, R. M. Cutri $^{3}$, J. Dailey ${ }^{3}$, P. R. M. EisenhardT $^{1}$, \\ R. S. McMillan ${ }^{4}$, T. B. Spahr ${ }^{5}$, M. F. Skrutskie ${ }^{6}$, D. Tholen ${ }^{7}$, R. G. Walker ${ }^{8}$, E. L. Wright ${ }^{9}$, E. DeBaun $^{1,10}$, \\ D. Elsbury ${ }^{1,11}$, T. Gautier IV ${ }^{1,12}$, S. Gomillion ${ }^{1,13}$, And A. Wilkins ${ }^{1,14}$ \\ ${ }^{1}$ Jet Propulsion Laboratory/California Institute of Technology, Pasadena, CA 91109, USA; Joseph.Masiero@jpl.nasa.gov \\ 2 Department of Physics and Astronomy, Johns Hopkins University, Baltimore, MD 21218, USA \\ ${ }^{3}$ Infrared Processing and Analysis Center, California Institute of Technology, Pasadena, CA 91125, USA \\ ${ }^{4}$ Lunar and Planetary Laboratory, University of Arizona, Kuiper Space Science Building 92, Tucson, AZ 85721-0092, USA \\ ${ }^{5}$ Minor Planet Center, Harvard-Smithsonian Center for Astrophysics, Cambridge, MA 02138, USA \\ ${ }^{6}$ Department of Astronomy, University of Virginia, Charlottesville, VA 22903-0818, USA \\ ${ }^{7}$ Institute for Astronomy, University of Hawaii, Honolulu, HI 96822, USA \\ ${ }^{8}$ Monterey Institute for Research in Astronomy, Monterey, CA, USA \\ ${ }^{9}$ UCLA Astronomy, Los Angeles, CA 90095-1547, USA \\ ${ }^{10}$ Dartmouth College, Hanover, NH 03755, USA \\ ${ }^{11}$ Notre Dame High School, Sherman Oaks, CA 91423, USA \\ ${ }^{12}$ Flintridge Preparatory School, La Canada, CA 91101, USA \\ ${ }^{13}$ Embry-Riddle Aeronautical University, Daytona Beach, FL 32114, USA \\ ${ }^{14}$ Department of Astronomy, University of Maryland, College Park, MD 20742, USA \\ Received 2011 May 3; accepted 2011 June 30; published 2011 October 17
}

\begin{abstract}
We present initial results from the Wide-field Infrared Survey Explorer (WISE), a four-band all-sky thermal infrared survey that produces data well suited for measuring the physical properties of asteroids, and the NEOWISE enhancement to the WISE mission allowing for detailed study of solar system objects. Using a NEATM thermal model fitting routine, we compute diameters for over 100,000 Main Belt asteroids from their IR thermal flux, with errors better than $10 \%$. We then incorporate literature values of visible measurements (in the form of the $H$ absolute magnitude) to determine albedos. Using these data we investigate the albedo and diameter distributions of the Main Belt. As observed previously, we find a change in the average albedo when comparing the inner, middle, and outer portions of the Main Belt. We also confirm that the albedo distribution of each region is strongly bimodal. We observe groupings of objects with similar albedos in regions of the Main Belt associated with dynamical breakup families. Asteroid families typically show a characteristic albedo for all members, but there are notable exceptions to this. This paper is the first look at the Main Belt asteroids in the WISE data, and only represents the preliminary, observed raw size, and albedo distributions for the populations considered. These distributions are subject to survey biases inherent to the NEOWISE data set and cannot yet be interpreted as describing the true populations; the debiased size and albedo distributions will be the subject of the next paper in this series.
\end{abstract}

Key words: infrared: general - minor planets, asteroids: general

Online-only material: color figures, machine-readable table

\section{INTRODUCTION}

Since the discovery of (1) Ceres in 1801 (Piazzi 1801) the majority of observations of asteroids and other minor planets have been conducted in visible wavelengths. While visible light can provide very accurate positions, the interpretation of photometry is rendered ambiguous by the dependence of the observed flux on both the size and albedo of the asteroid. This relationship is described by the equation

$$
D=\frac{1329}{\sqrt{p_{V}}} 10^{-H / 5}
$$

(see Harris \& Lagerros 2002 for an overview and references therein for its derivation), where $D$ is the diameter in kilometers, $p_{V}$ is the visible geometric albedo, and $H$ is the absolute magnitude which is defined as the apparent magnitude the body would have $1 \mathrm{AU}$ from the Sun, $1 \mathrm{AU}$ from the observer, and at $0^{\circ}$ phase angle. Albedos of solar system objects are observed to vary from only a few percent up to nearly $100 \%$ for icy surfaces, causing diameters inferred from visible data alone to have nearly an order of magnitude uncertainty. Multiwavelength visible surveys have found statistical correlations between the albedos and the visible colors of asteroids (e.g., Ivezić et al. 2001); however, the accuracy of the mapping between these two properties for individual objects is currently being examined (Mainzer et al. 2011c). Using an independent method of measurement for either the albedo or the diameter allows for the unique solution of the other, given a visible $H$ value.

Accurate diameters and albedos for a large number of asteroids enable a number of important areas of research into the history and formation of the solar system. Diameter measurements of asteroids based on infrared flux allow us to quantify the size-frequency distribution (SFD) of the bodies in a way independent of assumptions about the translation from $H$ magnitude to diameter that are typically required. Both the initial accretion and formation process of asteroids as well as the subsequent collisional and orbital evolution affect the current Main Belt SFD, and a well-measured SFD will allow us to put constraints on these processes. The albedo of an asteroid, meanwhile, is a strong function of its composition. Compositional gradients for the Main Belt have been shown in the past from both infrared and spectral surveys (e.g., Zellner 1979; Gradie \& Tedesco 1982; Tedesco et al. 2002; Bus \& Binzel 2002), but only for a limited number of objects. 
A large survey conducted in mid-infrared wavelengths will allow Main Belt albedos and diameters to be produced with good accuracy; this in turn will allow us to study the compositional gradient of the solar system and may ultimately allow us to set constraints on any major planetary migration that may have occurred.

Recent work has attempted to understand the SFD of asteroids as it relates to the impact physics dominating the evolution of the Main Belt with the aid of numerical simulations. O'Brien \& Greenberg (2003) analytically calculated the behavior of a population in a steady-state collisionally dominated regime to determine how the slope of a population's SFD behaved as a function of the mechanical strength of the material composing the body. They find that while a strengthless regime yields a nominal power-law slope of $a=-3.5$, including the strength of the body can vary the result over a range of slopes depending on the specific circumstances. Expanding on this, Durda et al. (2007) simulated a variety of impacts with numerical hydrocodes to look for the effect of internal structure, impact angle, impact velocity, and impactor-target size ratio on the resultant SFD of the shattered products. These authors have also attempted to match their numerical results to measured SFDs for asteroid families to determine the initial size of the parent body; however, the data used have depended on assumptions for the albedos of the asteroids. For the larger Main Belt population, Bottke et al. (2005a) have modeled the evolution of various initial size distributions to determine what the current Main Belt asteroid (MBA) SFD can indicate about the SFD present at the formation of the asteroids. Their results show a clear peak in formation SFD, with few objects smaller than $D \sim 100 \mathrm{~km}$ forming directly from the protosolar nebula; however, these results are also based on diameters determined from assumed albedos. Similarly, Bottke et al. (2005b) have investigated the sources and sinks of excited MBAs and the effect orbital evolution has on the SFD of the Main Belt.

Studies of asteroid albedo distributions have in the past been limited by small data sets. Early measurements were made using thermal infrared detectors on ground-based telescopes to determine radiometric diameters and thus albedos for tens of objects (e.g., Morrison 1974). The Infrared Astronomical Satellite (IRAS) revolutionized studies of physical properties of the Main Belt by observing over 2000 asteroids in the thermal infrared, determining albedos and diameters for these bodies in a uniform way (Tedesco et al. 2002, 2004). To date, IRAS represents the largest and most complete survey of asteroid albedos in the literature.

If the sizes and albedos of the members of an asteroid family are known, it is possible to use their orbital evolution to study their age. The Yarkovsky effect (see Bottke et al. 2006 for a review of the subject) occurs when incident optical light is absorbed and re-emitted as thermal infrared photons in a different direction, usually due to the rotation of the body. This difference in momentum causes changes in the orbit of the body over long time intervals. Nesvorný \& Bottke (2004) use this effect to refine predictions for the age of the Karin family based on backward integration of orbital parameters. As Karin is one of the youngest families (Nesvorný et al. 2002) this effect becomes increasingly important for accurate age dating of families the older they are. The accuracy of this technique, however, depends on knowing sizes and albedos of the objects to a high degree of certainty.

The distribution of albedos in the Main Belt as a whole and broken down by region can provide us with a window into the changing chemical and mineralogical processes active in different regions of the early solar system. Although the current understanding of the history of the Main Belt has the asteroids forming in or near their current locations, new theories are being proposed that the Main Belt may in fact be the result of a mixing of two distinct populations from different regions of the solar system. Migrations of the giant planets may have both cleared many of the objects that initially formed in the Main Belt region and repopulated this area with objects from beyond the "snow line" (Morbidelli et al. 2010; Walsh \& Morbidelli 2011). We then might expect the Main Belt to be composed of two overlapping populations, one having formed in a volatile-poor region of the protosolar disk and one forming in a volatile-rich area, though the latter population would lose any surface volatiles over the age of the solar system. Under this scenario albedo may be able to trace dynamical evolution as well as chemical processing.

There are very few ways to measure the albedo of an asteroid directly. Observations taken in situ by spacecraft can be used to measure both the absolute albedo and its variation across a body's surface (e.g., Howett et al. 2010), as well as its diameter, though only a handful of objects have been visited by spacecraft. Imaging polarimetry can also be used to determine the integrated surface albedo for a number of objects (Cellino et al. 1999), but appropriately calibrated instrumentation is uncommon. Similarly, direct measurements of asteroid diameters can come from a variety of techniques. Resolved imaging of an asteroid provides the simplest method of size determination; however, the use of, e.g., Hubble Space Telescope or Keck adaptive optics (AO) only allows the largest few asteroids to be resolved (e.g., Schmidt et al. 2009; Li et al. 2010). Asteroid occultations of background stars also provide a robust method of diameter measurement (Shevchenko \& Tedesco 2006); however, the logistical constraints of occultation events make obtaining a large sample difficult, and shape measurements are only instantaneous projections. Radar measurements can provide precise distances, rotation rates, projected profiles, and with sufficient data three-dimensional shape models (Ostro et al. 2002), but returned fluxes fall off quickly with distance, which limits the number of objects observable with this technique.

Indirect measurements of asteroid diameters and albedos can provide a wealth of information both for individual objects and the population as a whole. These techniques typically can observe a large number of objects in a relatively short period of time, provide uniform data for an entire population, and have understood biases that allow for determination of the true, underlying distributions. Careful calibration of indirect measurements is required, but once established these techniques can provide highly accurate measurements of asteroid physical properties for a large number of bodies.

For objects that have known orbits, measurement of the infrared flux emitted from the surface can be used to constrain the diameter of the body (see Section 3 for a discussion of the method used here). Infrared imaging can be accomplished rapidly when integrated in an all-sky survey. Thermal infrared measurements of a large sample of asteroids represent the best way to determine robust diameters rapidly for many thousands of objects. In this paper, we present preliminary results from the Wide-field Infrared Survey Explorer (WISE) space telescope, the next-generation all-sky infrared survey, focusing here on the cryogenic observations of MBAs. 


\section{OBSERVATIONS}

\subsection{WISE and NEOWISE}

Launched on 2009 December 14, WISE is a thermal infrared space telescope that performed an all-sky survey from 2010 January 14 until it exhausted the telescope-cooling hydrogen ice on 2010 August 5. The survey continued during the warmup of this secondary tank with limited sensitivity in the longer wavelengths until the primary coolant, responsible for maintaining detector temperature, was exhausted on 2010 October 1. WISE subsequently entered a Post-Cryogenic Mission to complete the survey of the largest MBAs, continue discovering new near-Earth objects (NEOs), and complete a second-pass survey of the inertial sky in the two shortest wavelengths. During its cryogenic mission, WISE imaged the sky in four infrared wavelengths simultaneously using dichroic beam splitters to produce co-boresight images with band centers at $3.4 \mu \mathrm{m}, 4.6 \mu \mathrm{m}$, $12 \mu \mathrm{m}$, and $22 \mu \mathrm{m}$ ( $W 1, W 2, W 3$, and $W 4$, respectively). The latter two bands are particularly important for solar system studies as the dominant amount of the flux received from asteroids is from thermal emission peaking at these wavelengths. First-pass calibration of the WISE data was tuned to the fully cryogenic mission and while the final calibration currently being undertaken will finalize measurements obtained during each warmup stage, we restrict our current analysis to objects observed during this fully cryogenic stage. Prelaunch descriptions of WISE were given by Mainzer et al. (2006) and Liu et al. (2008), while postlaunch overviews, including initial calibrations and color corrections, are presented by Wright et al. (2010) and Mainzer et al. (2011b).

The WISE survey follows a continuous scan along lines of ecliptic longitude at a solar elongation of $\sim 90^{\circ}$ as the spacecraft orbits above the terminator of the Earth. An oscillating scan mirror compensates for this motion, providing stable images with effective integration times of $8.8 \mathrm{~s}$. The positions of all known minor planets are propagated to the time of each observation and checked against all transient sources in the field of view, recording the appropriate calibrated magnitudes if observed. In this way, thermal measurements of each minor planet cataloged by the Minor Planet Center (MPC) ${ }^{15}$ can be found. In addition to previously known objects, the WISE processing pipeline includes the capability to discover new objects via the NEOWISE enhancement (Mainzer et al. 2011a). While comprehensive follow-up of new potential NEOs has been one of the priorities of the WISE team (A. K. Mainzer et al. 2011, in preparation), the large number of Main Belt discoveries $(>34,000)$ has prohibited sufficient immediate ground-based follow-up. Largescale asteroid surveys such as Spacewatch and the Catalina Sky Survey have already provided serendipitous follow-up of many of the new WISE MBAs and will continue to do so, while future surveys are expected to recover the remainder. As described in Wright et al. (2010) and Mainzer et al. (2011a), the WISE cadence resulted in an average of 10-12 observations of each minor planet detected over $\sim 36 \mathrm{hr}$. For a subset of the Main Belt, predominantly in the outer regions, observations were obtained at two or more epochs depending on the relative motion of the object and Earth around the Sun.

\footnotetext{
15 See the MPCORB.DAT file available here:

http://www.minorplanetcenter.net/iau/MPCORB.html.
}

\subsection{Data and Calibration}

In this paper, we consider only those MBAs detected by NEOWISE/WMOPS during the cryogenic portion of the mission, shown in a top-down view of the inner solar system in Figure 1. This consists of 129,750 unique objects. We obtained our data used for fitting in a method identical to the one described in Mainzer et al. (2011b) and A. K. Mainzer et al. (2011, in preparation), though tuned for MBAs. Specifically, we queried the MPC observation file ${ }^{16}$ for all observations submitted from WISE (observatory code C51). We then used the resultant R.A.-decl.-time values as input for a query of the WISE individual exposure archive, the "Level 1b" data, through the Gator tool provided by the Infrared Science Archive (IRSA ${ }^{17}$ ). To ensure that only the observations of the moving objects were returned, we restricted our search radius to 0 '. 3 from the position and $2 \mathrm{~s}$ from the time obtained for the detection from the MPC. Additionally, we set a constraint of JD $<2,455,413.5$ to ensure that only data during the fully cryogenic mission was used for this initial survey. This method of data acquisition has the benefit of ensuring all observations used have been vetted both internally by the WISE data pipeline and again by the MPC.

All data were processed using the first-pass version of the WISE pipeline, which computed dark current/sky offset levels, flagged instrumental artifacts such as latent images and diffraction spikes and performed linearity compensation. Only observations with an artifact flag cc_flags $=0$ or $p$ in a band were accepted: a value of 0 indicates no evidence of artifact was found by the pipeline, while $p$ indicates the possibility of contamination by a latent image. As discussed in Mainzer et al. (2011b), we find that the pipeline was overly conservative in artifact flagging and cc_flags $=p$ values have similar fluxes to cc_flags $=0$ detections while increasing the number of usable observations by $\sim 20 \%$. The ph_qual flag was required to have a value of $\mathrm{A}, \mathrm{B}$, or $\mathrm{C}$ to again ensure only valid detections were used. Nonlinearity and saturation are a particular concern for the brightest MBAs, especially in bands $W 3$ and $W 4$. The WISE data reduction pipeline applies a nonlinearity and saturation correction for all observations brighter than the threshold of $W 1=7.8 \mathrm{mag}, W 2=6.5 \mathrm{mag}$, $W 3=3.6 \mathrm{mag}$, and $W 4=-0.6 \mathrm{mag}$. Objects with magnitudes brighter than $W 3=4$ and $W 4=3$ were assigned errors of 0.2 mag to account for the change in the point-spread function for very bright objects, and a linear correction to the magnitudes of sources with $-2<W 3<4$ was applied (Mainzer et al. 2011b; Cutri et al. 2011). Following those authors, we did not use objects brighter than $W 3=-2$ and $W 4=-6$ for thermal modeling.

Each object was required to have been observed at least three times in one WISE band with magnitude error $\sigma_{\text {mag }} \leqslant$ 0.25 to undergo thermal modeling, as a precaution against contamination by spurious sources (e.g., background noise, cosmic rays, stars, etc.). For multiple-band thermal models we required other bands to have at least $40 \%$ of the detection rate of the band with the largest number of detections, usually W3 for MBAs. To reduce the possibility of confusion with inertially fixed sources such as stars and galaxies, we searched each position retrieved from the Level $1 \mathrm{~b}$ catalog in the Daily and Atlas Coadded Catalogs (also served by IRSA) within 6.5 , equivalent to the $W 1, W 2$, and $W 3$ beam sizes. These

\footnotetext{
16

http://www.minorplanetcenter.net/iau/ECS/MPCAT-OBS/MPCAT-OBS.html

$17 \mathrm{http} / /$ irsa.ipac.caltech.edu/applications/Gator/
} 


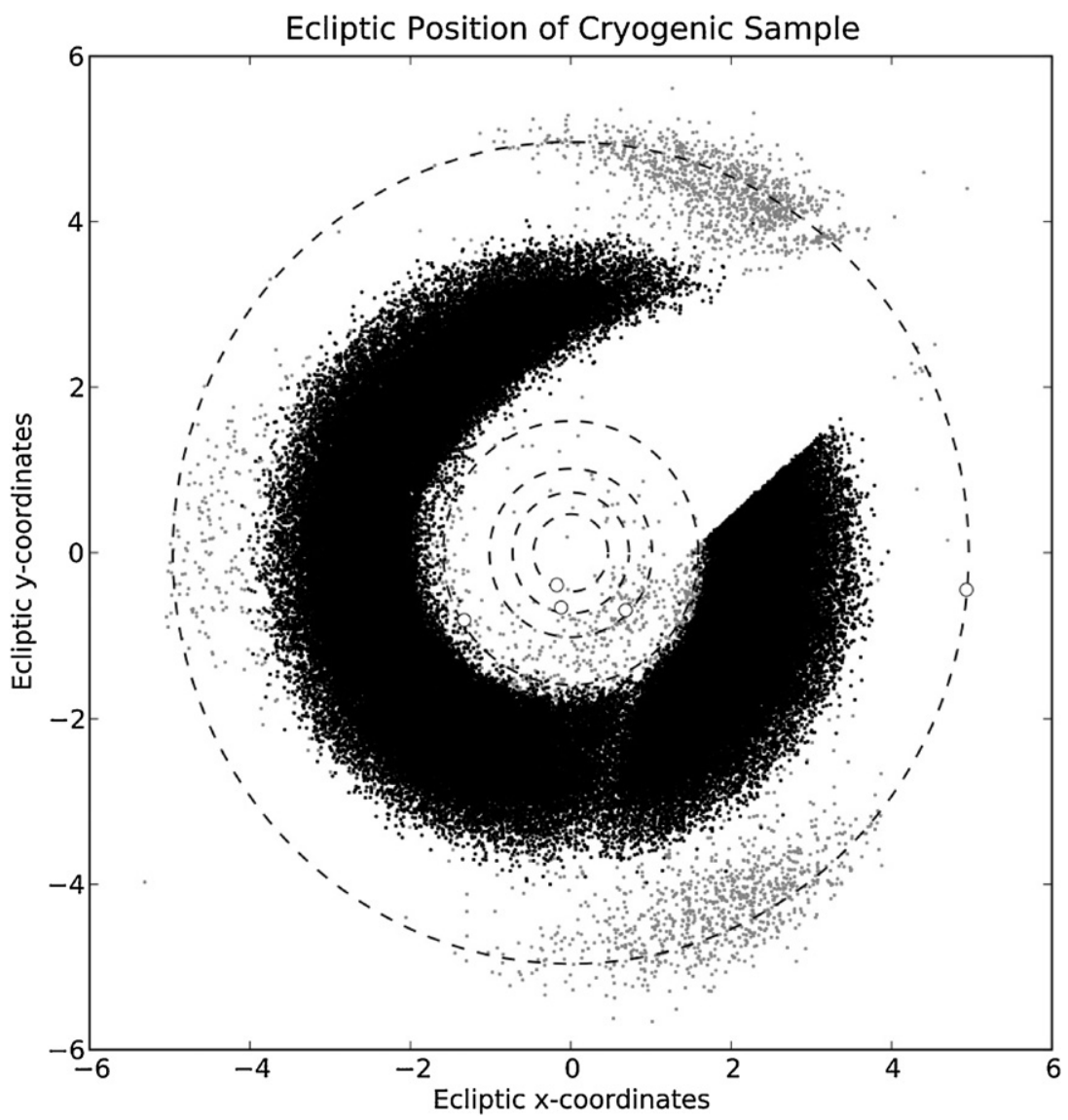

Figure 1. Top-down view of the inner solar system showing the location of all objects observed during the fully cryogenic mission. Positions were propagated to 2010 August 5, the date of the exhaustion of coolant from the secondary tank. Black points indicate MBAs while gray points are all other solar system objects. Axes' units are AU.

searches looked for sources that appeared at least twice and in at least $30 \%$ of the images covering that location. Any sources returned from these searches were considered to be inertial, which could contaminate the observation of the asteroid at that position. Thus that asteroid detection was discarded from the thermal modeling routine.

In Figure 2, we show the mean colors of inner solar system objects as observed during the cryogenic phase of the WISE mission with the MBAs highlighted in black (see A.K. Mainzer et al. 2011, in preparation; Grav et al. 2011, for discussions on the other populations shown in this figure). The bifurcation in the $W 1-W 2$ color observed for the MBAs traces the two dominant albedo groupings in the Main Belt (see below for further discussion). The MBAs span a wide range of colors and are bounded by the NEO and Jupiter Trojan populations. Note that MBAs occupying color-space typically associated with one of the other populations are candidates for objects that may have been misidentified as MBAs during preliminary orbit fitting. We show in Figure 3 the color for each object as a function of heliocentric distance at the time of observation. Color, especially in bands dominated by thermal emission, is a strong function of temperature of the body and thus distance from the Sun. Figure 4 shows the sky-plane velocity for each object compared with its $W 3-W 4$ color. The mean sky-plane velocity for MBAs is 0.2 day $^{-1}$; objects with velocities significantly larger than this are candidates for NEOs misclassified as MBAs, requiring further follow-up. Future work will combine color and skyplane velocity cuts to distinguish MBAs with poorly known orbits from NEOs and Trojans.

\section{DIAMETER AND ALBEDO DETERMINATION THROUGH THERMAL MODELING}

In contrast to the visible flux received from an asteroid, which is reflected sunlight, the mid-IR flux beyond $\sim 6 \mu \mathrm{m}$ from an object in the Main Belt is almost completely thermal emission from that body. For an object with an established orbit, the phase angle and distances to the asteroid from the Earth and the Sun are well known; thus the observed thermal flux can be converted into a total emitted flux at the surface. By making assumptions for some physical surface properties, a diameter can be computed from a single band detection. When simultaneous thermal measurements at multiple wavelengths are available the beaming parameter of the surface material may also be fit to the data. The beaming parameter $(\eta)$ represents the deviation of the thermal emission from that of a smooth non-rotating sphere due to rotation and surface roughness, and is used to consolidate the uncertainty in the values of the surface thermal properties, including emissivity. When visible-light data are additionally available, the visible albedo $\left(p_{V}\right)$ becomes a free parameter that can now also be fit using the two data sets in conjunction. Further data such as $W 1$ or $W 2$ measurements that are dominated by reflected sunlight allow us to derive an independent measure of the ratio of visible to near-infrared (NIR) reflectance as well.

In order to analyze the thermal infrared asteroid measurements from the IRAS satellite, Lebofsky et al. (1986) developed the "Standard Thermal Model" (STM) for asteroids, calibrated against measurements from (1) Ceres and (2) Pallas. 

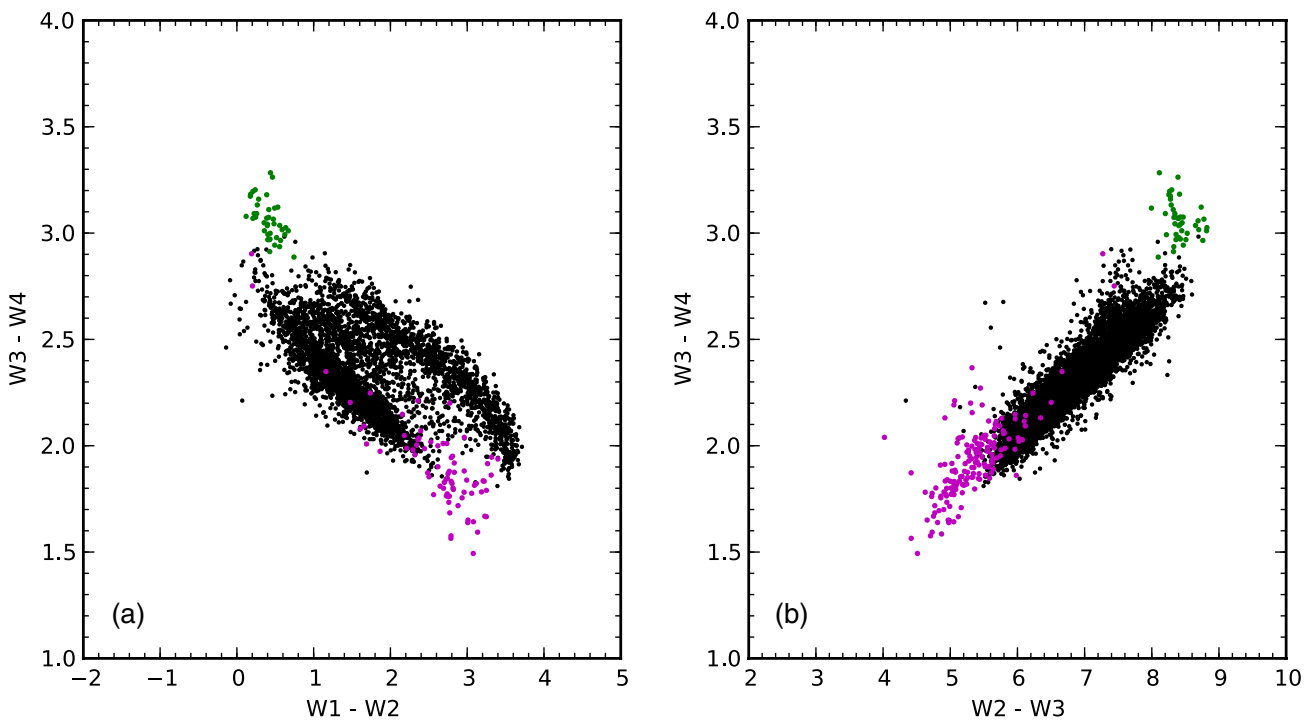

Figure 2. (a) $W 1-W 2$ vs. $W 3-W 4$ colors for all MBAs (black), Trojans (green), and NEOs (magenta); (b) $W 2-W 3$ vs. $W 3-W 4$ colors for these same populations. (A color version of this figure is available in the online journal.)
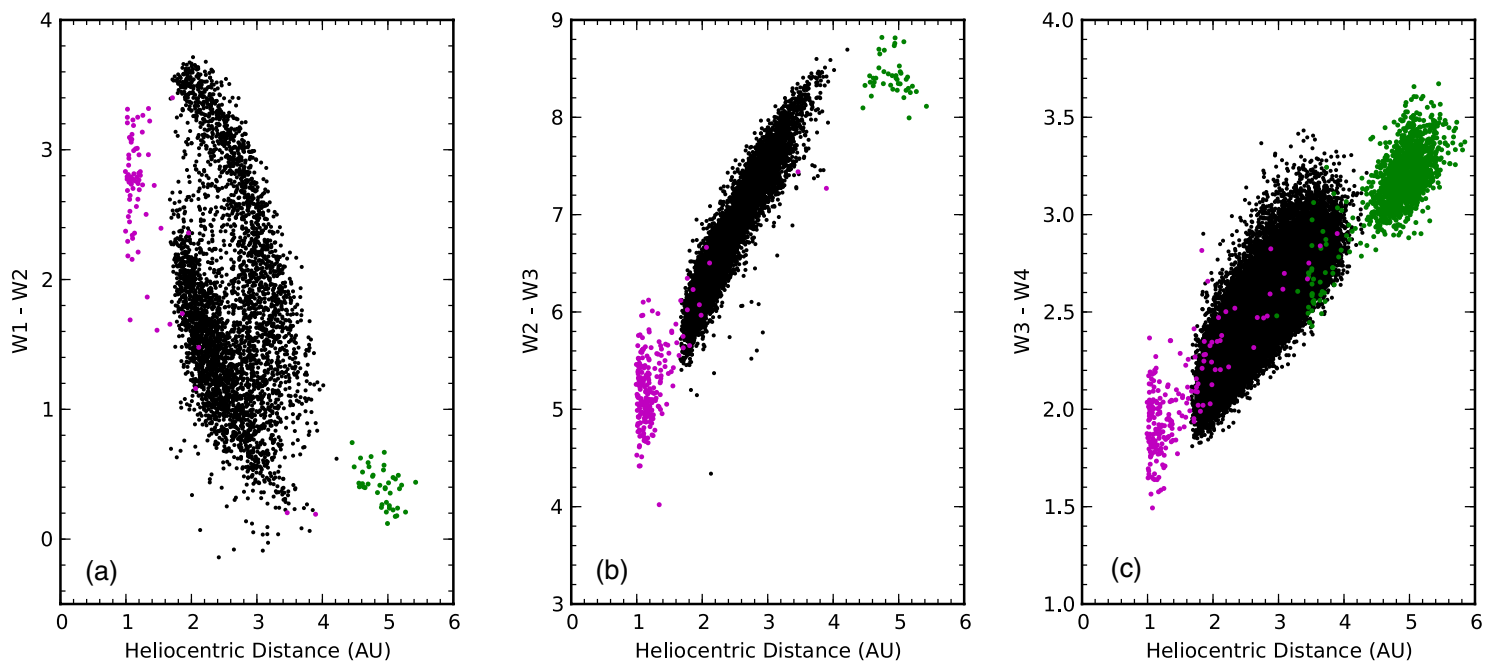

Figure 3. (a) $W 1-W 2$ vs. heliocentric distance for all MBAs (black), Trojans (green), and NEOs (magenta); (b) as in (a) but for W2-W3 color; (c) as in (a) but for W3-W4 color.

(A color version of this figure is available in the online journal.)

In this model, the beaming parameter was held constant to $\eta=0.756$ based on the ground-truth occultation observations of the calibrator asteroids. Subsequent work indicated that this model might not be appropriate for smaller asteroids, thus Harris (1998) modified STM to a form appropriate for a "NearEarth Asteroid Thermal Model" (NEATM), where $\eta$ is allowed to vary. While designed specifically to account for the breakdown of STM when considering NEOs, NEATM can be applied quite readily to a wide range of bodies in the solar system (e.g., Mueller et al. 2010; Ryan \& Woodward 2010). Wright (2007) compared NEATM with a full thermophysical model of a cratered surface and found that for low phases both models produce consistent results. Lebofsky \& Spencer (1989) present an investigation of objects with a beaming parameter at the theoretical maximum of $\eta=\pi$, which occurs for a body rotating so quickly it is latitudinally isothermal.

We have performed preliminary thermal modeling of MBAs based on the WISE First-Pass Data Processing Pipeline described above, covering observations taken during the cryogenic phase of the mission. We modeled each object as a non-rotating sphere with triangular facets and variable diameter, beaming parameter, visible albedo, and NIR reflectance ratio as appropriate for the data. Relative distances and phase angles were computed for each measurement to ensure changing distances do not bias the resultant fits. Although we do not expect all, or even most, asteroids to have a spherical shape, our observations covering $\sim 36 \mathrm{hr}$ smooth out rotation effects and allow us to determine the effective diameter of a spherical body with the same physical properties. Long period rotators $(P \sim$ days $)$ with large amplitudes, e.g., binary asteroids with mass fraction $\sim 1$, will have poor fits resulting in a moderate mismeasurement of albedo and diameter. Future work will address the light-curve component of our data set to determine the minimum and maximum sizes of our targets in order to estimate first-order shape models as well as estimates of the fraction of binaries in the Main Belt.

At each instance, the temperature on every facet was computed and color corrected based on the values in Wright et al. (2010). The emitted thermal flux for each facet was computed with the NEATM model and nightside facets were assumed to contribute zero flux. A reflected light model was used to 

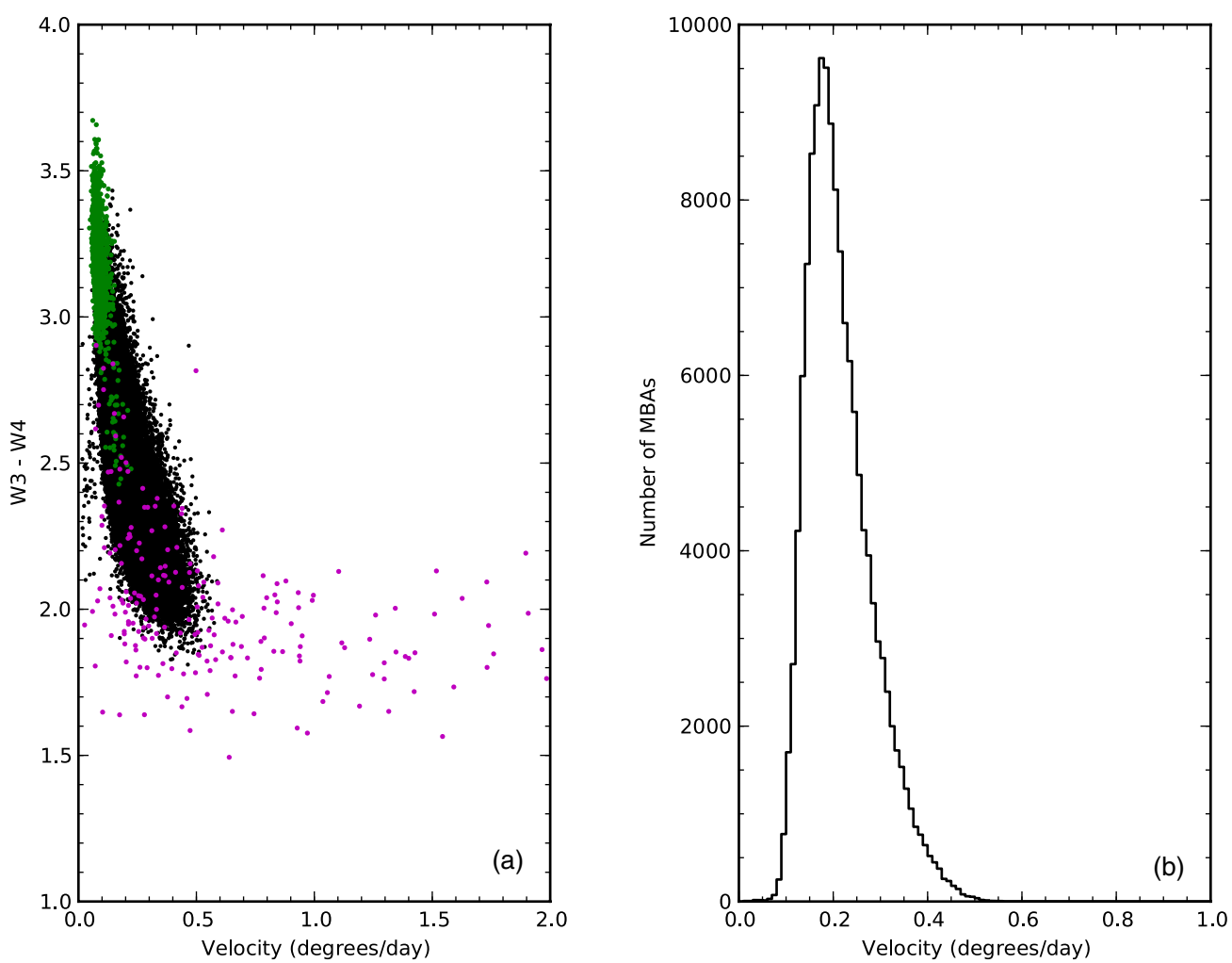

Figure 4. (a) W3-W4 color vs. sky-plane velocity for all MBAs (black), Trojans (green), and NEOs (magenta); (b) differential distribution of sky-plane velocities for all MBAs.

(A color version of this figure is available in the online journal.)

determine the reflected component in each band for all illuminated facets visible to WISE at the time of observation; this was most important for $W 1$ and $W 2$. The model reflected and emitted light was summed for all facets and converted to a model magnitude using the Jansky flux of a zero magnitude source provided in Wright et al. (2010) and modified according to the text for red sources: $306.681 \mathrm{Jy}$ for $W 1,170.663 \mathrm{Jy}$ for $W 2,31.3684$ Jy for $W 3$, and $7.9525 \mathrm{Jy}$ for $W 4$. Note that the modifications in the $W 3$ and $W 4$ zero points are the result of adjusting the central wavelengths of these bands to $\lambda_{0, W 3}=11.0984 \mu \mathrm{m}$ and $\lambda_{0, W 4}=22.6405 \mu \mathrm{m}$ to correct the discrepancy observed in the calibration tests between blue-spectrum and red-spectrum objects. These model magnitudes were then compared with the measured magnitudes, and the model was iterated through a least-squares fitting routine until the best fit was found.

For objects with two thermally dominated bands, the beaming parameter was allowed to vary, while for those with only one thermal band we used a fixed value of $\eta=1.0$, based on the peak of the $\eta$ distribution of MBAs that were fit with a variable beaming parameter (see below). For objects where no detected band was dominated $(>75 \%)$ by reflected light we assumed an NIR reflectance ratio of 1.5 as was found for MBAs with fitted NIR reflectances (see below). These objects typically were not detected in $W 1$ and either were not detected or had both thermal emission and reflected light in $W 2$.

Monte Carlo (MC) simulations were performed in each case to determine the errors on all variable parameters. For high signal-to-noise ratio cases where the quoted error on the measured magnitude only represented the statistical error, we set a floor of $\sigma_{\text {mag }}=0.03$, representing the absolute error on the photometry (Wright et al. 2010). For all objects we assumed that the emissivity $\epsilon=0.9$ and we assumed that the magnitude- phase slope parameter (cf. Bowell et al. 1989) was $G=0.15$ unless otherwise given by the MPC or in the Lightcurve Database (LCDB ${ }^{18}$; Warner et al. 2009a). The quoted errors on the modeled parameters are equal to the weighted standard deviation of all MC trial values. For objects with a fixed beaming parameter, an error of $\sigma_{\eta}=0.2$ was assumed to allow for proper error determination of derived parameters based on the mean and standard deviation of all best-fitting beaming parameters for objects with fitted values (see Section 5). Similarly, for objects with fixed IR reflectance ratios, we assume an error bar of $\sigma_{\text {ratio }}=0.5$ based on the mean and standard deviation of objects with fitted IR reflectance ratios (see Section 8). We note that the flux calibrations presented by Mainzer et al. (2011b) set a lower limit on the accuracy of computed diameters for sources in the WISE data of $\sigma_{D}=10 \%$. This error implies a minimum fractional error for albedo of $\sigma_{p_{V}}=20 \% \times p_{V}$ assuming a perfect $H$ magnitude. These values are in addition to any Poissonian error inherent to the observations, though for most of the objects presented here the calibration errors dominate our solutions.

In total 129,750 MBAs, selected from the cryogenic phase of the survey, had a sufficient number and quality of detections to allow us to perform thermal modeling and determine their effective diameter. Of these, 17,482 objects had orbital arcs shorter than 30 days; as such their orbits have a larger uncertainty than the rest of the population, which corresponds to uncertainty in their geocentric and heliocentric distances, which will naturally increase the error on their calculated diameters. Additionally, 112,265 objects also had available optical data allowing us to calculate albedo as well as diameter. Both of these latter two

\footnotetext{
18 http://www.minorplanet.info/lightcurvedatabase.html
} 
Table 1

Example of Electronic Table of the Thermal Model Fits

\begin{tabular}{|c|c|c|c|c|c|c|c|}
\hline Object & $\mathrm{H}$ & $\mathrm{G}$ & $\mathrm{D}$ & $p_{V}$ & $\eta$ & $p_{I R}$ & \# obs (W1-W4) \\
\hline 00002 & 4.060 & 0.11 & $544.000 \pm 42.916$ & $0.1417 \pm 0.0195$ & $0.938 \pm 0.049$ & $0.0961 \pm 0.0229$ & 1111110 \\
\hline 00002 & 4.060 & 0.11 & $544.000 \pm 60.714$ & $0.1419 \pm 0.0456$ & $0.774 \pm 0.074$ & $0.0816 \pm 0.0155$ & 111180 \\
\hline 00005 & 6.850 & 0.15 & $115.000 \pm 9.353$ & $0.2451 \pm 0.0509$ & $0.994 \pm 0.065$ & $0.3313 \pm 0.0669$ & 9131313 \\
\hline 00006 & 5.710 & 0.24 & $185.000 \pm 10.688$ & $0.2685 \pm 0.0488$ & $0.840 \pm 0.061$ & $0.3810 \pm 0.0346$ & 14141413 \\
\hline 00008 & 6.350 & 0.28 & $140.000 \pm 1.160$ & $0.2614 \pm 0.0484$ & $0.794 \pm 0.029$ & $0.4348 \pm 0.0453$ & 15171617 \\
\hline 00009 & 6.280 & 0.17 & $204.528 \pm 3.671$ & $0.1300 \pm 0.0184$ & $1.059 \pm 0.012$ & $0.2665 \pm 0.0366$ & 15151415 \\
\hline 00009 & 6.280 & 0.17 & $190.791 \pm 4.901$ & $0.1493 \pm 0.0343$ & $0.878 \pm 0.026$ & $0.3144 \pm 0.0188$ & 10101010 \\
\hline 00010 & 5.430 & 0.15 & $453.239 \pm 19.244$ & $0.0579 \pm 0.0051$ & $0.928 \pm 0.026$ & $0.0648 \pm 0.0054$ & 111160 \\
\hline 00011 & 6.610 & 0.15 & $159.108 \pm 5.944$ & $0.1585 \pm 0.0365$ & $0.937 \pm 0.048$ & $0.2923 \pm 0.0298$ & 910910 \\
\hline 00012 & 7.240 & 0.22 & $126.643 \pm 3.199$ & $0.1400 \pm 0.0137$ & $0.947 \pm 0.026$ & $0.2818 \pm 0.0341$ & 17191421 \\
\hline 00013 & 6.740 & 0.15 & $227.000 \pm 25.948$ & $0.0690 \pm 0.0218$ & $0.894 \pm 0.139$ & $0.0443 \pm 0.1426$ & 0448 \\
\hline
\end{tabular}

(This table is available in its entirety in a machine-readable form in the online journal. A portion is shown here for guidance regarding its form and content.)

populations are changing continuously, as ground-based surveys submit serendipitous visible observations of NEOWISEdiscovered asteroids (allowing us to fit albedos and allowing the MPC to fit better orbits), and as the MPC links WISE observations with previous one-night stands and lost asteroids.

We provide the full table of our best fits for MBAs from the Pass 1 processed cryogenic survey data in the online version of the journal, or online at: http://wise2.ipac.caltech.edu/staff/ bauer/NEOWISE_pass1/. A sample of the table is shown in Table 1. This table contains: the MPC-packed format name of the object; the $H$ and $G$ values used; the diameter, albedo, beaming parameter, and infrared albedo as well as associated error bars; and the number of observations in each WISE band that were used for fitting. For objects observed at multiple epochs with fits consistent across all observations, each epoch is presented as a separate row in the table. As discussed in Section 10, objects where multiple epochs were forced to fit to a single model because the separate fits were divergent are presented as a single row. Objects without optical data at the time of publication have "nan" ("not a number") values or -9.99 for absolute magnitude and albedo. Similarly, objects for which an infrared albedo could neither be fit nor had literature optical data that could be used with an assumed reflectance ratio to estimate an infrared albedo (see Section 8) have "nan" values or -9.99 in this entry.

\section{PRELIMINARY RAW SIZE-FREQUENCY DISTRIBUTION OF MBAs}

Using fluxes from the WISE data, and a faceted NEATM model, we are able to determine diameters for our observed objects. In Figure 5 we show the cumulative preliminary raw size-frequency distribution (PRSFD) for the three major regions of the Main Belt: the inner-Main Belt (IMB, those objects with 1.8 AU $<a<2.5 \mathrm{AU}$ ), the middle-Main Belt (MMB, objects with $2.5 \mathrm{AU}<a<2.82 \mathrm{AU}$ ), and the outer-Main Belt (OMB, objects with $2.82 \mathrm{AU}<a<3.6 \mathrm{AU}$ ). In all cases, perihelion distance was required to be beyond the orbit of Mars, $q>1.666$ AU. Also plotted on the distribution histograms are one-hundred-trial MC simulations of the diameter distribution including the appropriate measured errors. This mean MC distribution and associated error are shown as points; the error bar sizes are smaller than the point size. We see no significant change between the MC distribution from the distribution of best-fit diameters. We find the slope of the PRSFD for smaller objects in all three subpopulations to be consistent with the $a=-2.5$ value found by Gladman et al. (2009); however, debiasing will be critical to determining the true value of this

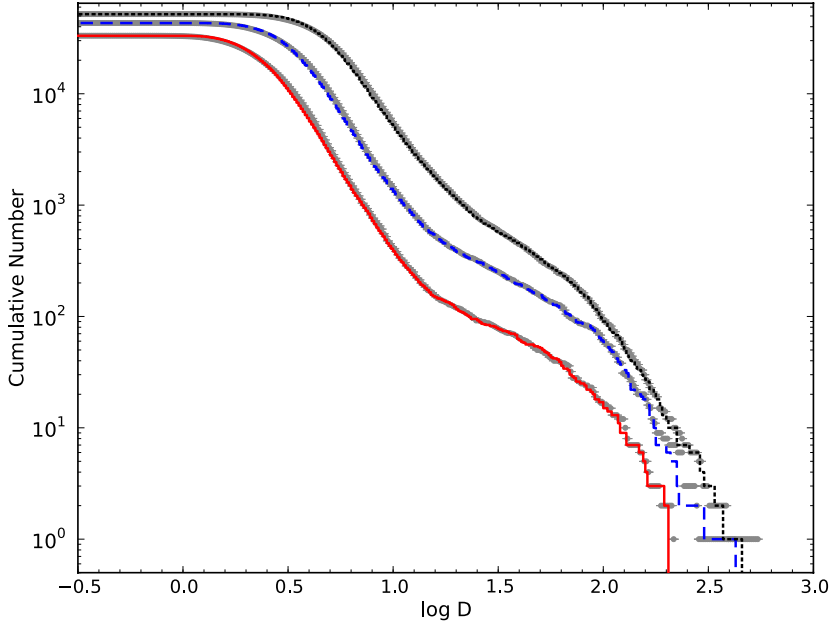

Figure 5. Cumulative raw size-frequency distribution of MBAs in the inner(red), middle- (blue), and outer-Main Belt (black). Plotted under the distributions are gray points showing the Monte Carlo simulation for each data set; the error bars are the size of the points.

(A color version of this figure is available in the online journal.)

slope. We observe a significant change in slope for the PRSFD between 15-25 km, consistent with the location of the "kink" seen in the debiased $H$ distribution by Jedicke \& Metcalfe (1998). A debiasing campaign of the observed population currently underway will allow us to explore the true SFDs of the populations and will be discussed in future work.

\section{VARIABLE BEAMING PARAMETERS}

For objects with detections in at least two thermal bands, we allow the beaming parameter to vary during the thermal model fitting. We are able to fit beaming parameters for 66,406 MBAs. We find a wide range of best-fit beaming parameters between the theoretical limits of 0.3 and $\pi$, with a peak value of $\eta=1.0$ and standard deviation of $\sigma_{\eta}=0.2$. In Figure 6 we show the beaming parameter found for all objects with fitted values as a function of a variety of orbital and physical parameters. Beaming parameter has a weak dependence on the semimajor axis (Figure 6(a)), an effect that is more pronounced for the relationship with phase angle (Figure 6(g)). Note that due to the constraints of the pointing of WISE over the course of the survey to solar elongations $\approx 90^{\circ}$, phase angle, heliocentric distance, and geocentric distance are strongly correlated, though ecliptic latitude of the observations as well small changes in the exact pointing over the survey weaken 

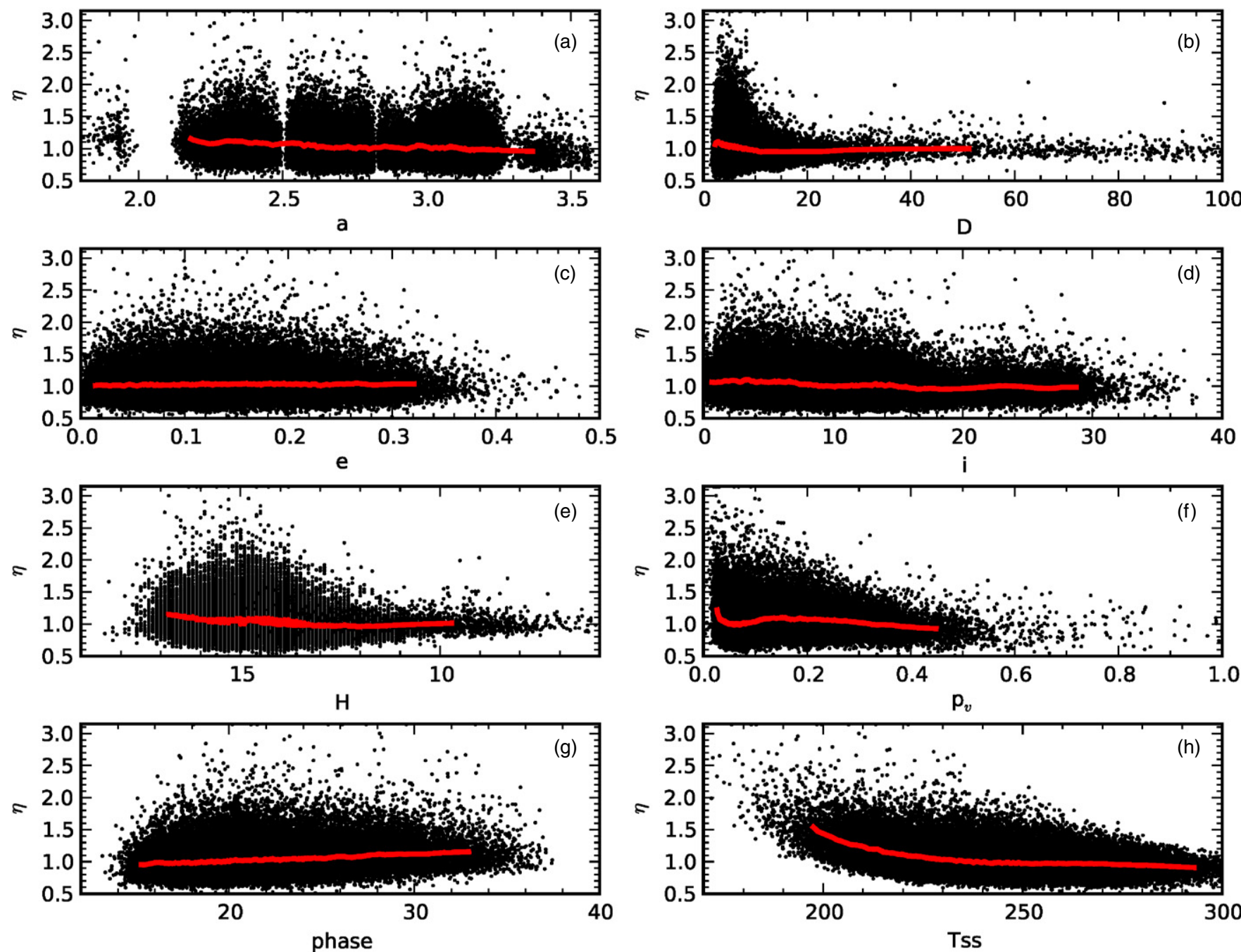

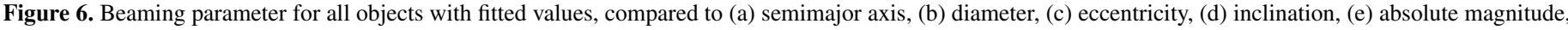

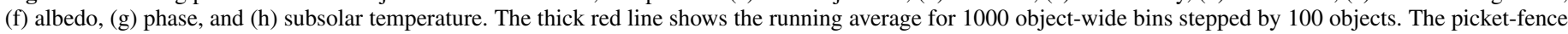
effect in the absolute magnitude is an artifact of the 0.1 mag reported precision of $H$ for most objects.

(A color version of this figure is available in the online journal.)

this relation. Wright (2007) has shown that for the phase angles we typically observe MBAs at $\left(14^{\circ} \lesssim \alpha \lesssim 27^{\circ}\right)$, the differences in calculated diameter and beaming parameter between NEATM and more realistic thermophysical models is minimal over a large range of observing geometries. From the raw distribution, the beaming parameter shows no dependence on size (Figure 6(b)), eccentricity (Figure 6(c)), inclination (Figure 6(d)), or absolute magnitude (Figure 6(e)).

We find a best-fit linear relation to the running average of beaming parameter as a function of phase of

$$
\eta=(0.79 \pm 0.01)+\alpha(0.011+0.001)
$$

where $\eta$ is the beaming parameter and $\alpha$ is the phase angle in degrees. This is consistent with the results found in A. K. Mainzer et al. (2011, in preparation) for the NEOs from WISE, but differs significantly from the results of Wolters \& Green (2009) who found a best-fitting line of $\eta=1.08+0.007 \alpha$. For phase angles within the Main Belt $\left(14^{\circ}-32^{\circ}\right)$ the average beaming ranges from $0.94<\eta<1.14$ although the spread around this value is large. Thus, $\eta_{\text {assumed }}=1.0$ for objects with only a single thermal band is a reasonable assumption for objects in the Main Belt. Debiasing will allow us to account for any detection-limit effects that may bias the selection of objects that have sufficient data for fitting of the beaming parameter.

Note that while the running average over the beaming parameter shows a dependence on albedo (Figure 6(f)) and subsolar temperature (Figure 6(h)), albedo determinations are very sensitive to survey biases, both from WISE and optical follow-up, while subsolar temperature is a function of beaming parameter as per the equation from the definition of NEATM in Harris (1998):

$$
T_{\mathrm{SS}}=[(1-A) S /(\eta \epsilon \sigma)]^{0.25}
$$

(where $T_{\mathrm{SS}}$ is the subsolar temperature, $A$ is the Bond albedo, $S$ is the incident solar flux, $\eta$ is the beaming parameter, $\epsilon$ is the emissivity, and $\sigma$ is the Stefan-Boltzmann constant), and so this cannot be used as an independent constraint.

We show in Figure 7 histograms of the beaming parameter distribution for the inner-, middle-, and outer-Main Belt populations. Also shown as points are the mean distribution and error from a one-hundred-trial MC simulation of the distribution using the error bar on each fitted beaming parameter. All populations show longer tails toward higher values of beaming parameter than toward lower, and have consistent shapes. 


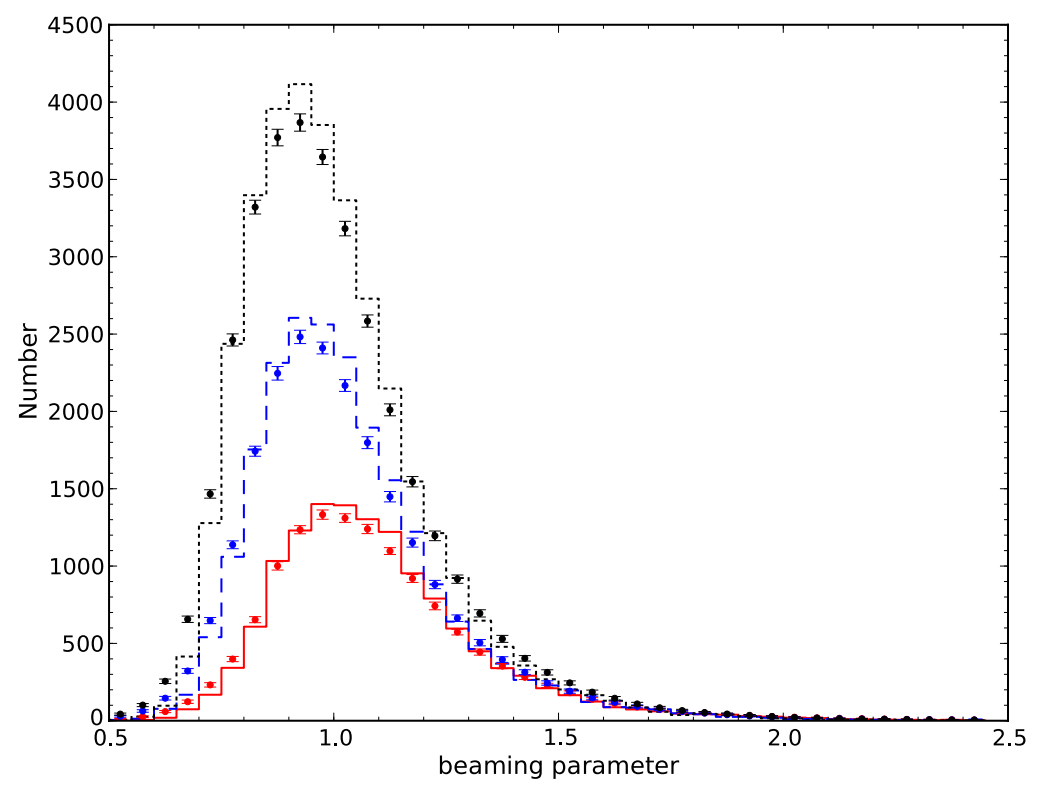

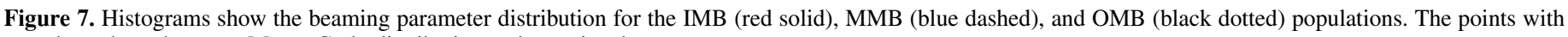
error bars show the mean Monte Carlo distribution and associated error.

(A color version of this figure is available in the online journal.)

The shift in peak beaming parameter with phase can be seen as the change in distribution between populations with different average phase angles.

\section{PRELIMINARY RAW ALBEDO DISTRIBUTION OF MBAs}

With the inclusion of visible data in our modeling, we determine albedos as well as diameters for the asteroids discussed here. We use the published $H$ and $G$ values for all asteroids, available from the MPC. During the confirmation of the calibration of WISE for asteroids, Mainzer et al. (2011b) investigated the need for an offset in $H$ to account for systematic errors in $H$ values, but found that no offset was required (cf. Jurić et al. 2002, who found a $0.2 \mathrm{mag}$ shift). The $H$ magnitudes were assigned a random error of $\sim 0.2 \mathrm{mag}$. We perform MC simulations of our visible light measurements as well as of the thermal measurements to quantify the error on albedo; however, in all cases the minimum error on albedo will be $20 \%$ (Mainzer et al. $2011 b$ ) for objects with optical data and one good thermal band. We have sufficient optical data to determine albedos for 112,265 MBAs. Though our individual albedos have large error bars, the population statistics can still provide us with a window into the state of the surface composition of the Main Belt.

In Figure 8 we show the differential preliminary raw albedo distribution (PRAD) of all the inner-, middle-, and outer-MBAs in our survey. We then take the fitted albedos and their respective error bars and perform a one-hundred-trial MC simulation of these values to find a mean distribution with errors, shown as points. In all cases, the peaks of the distributions broaden slightly in the MC simulation, which is expected. In log-albedo space the differential distribution is described well by a bimodal Gaussian distribution. We show our best-fitting double Gaussian (fitted to the mean distribution found through the MC simulations) as the smooth dotted curve under each set of points.

The bimodality in albedo likely traces the difference between the two major branches of asteroid composition: the S-type asteroids with high albedos and the C-type asteroids with low albedos (Chapman et al. 1975; Tedesco et al. 1989). Mainzer et al. (2011c) investigate the specific link between albedo and a variety of taxonomic classification systems. As discussed above, however, the PRAD will naturally include the observational biases of the ground-based telescopes used to determine the optical magnitudes needed to find the visible albedo, favoring higher albedo asteroids and over-representing their contribution to the total population, particularly in the IMB. Debiasing, currently being undertaken, will allow us to quantify and remove this effect.

The mean value and width of the Gaussian that best describes the dark peak of the PRAD for each population is consistent across populations, with mean albedo $\mu=0.06$ and a dispersion of $\sigma={ }_{-.02}^{+.03}$. Note that the Gaussian error bars on the $\mu$ value are in log space, and thus asymmetric in native units. Unlike the dark asteroids, the bright complex shows a distinct change in the mean value in the PRAD as one moves out in the Main Belt. The mean albedo of the bright peak for the Gaussian describing each population is: $\mu_{\mathrm{IMB}}=0.28, \mu_{\mathrm{MMB}}=0.25$, and $\mu_{\mathrm{OMB}}=0.17$, with widths of $\sigma_{\mathrm{IMB}}=_{-.09}^{+.13}, \sigma_{\mathrm{MMB}}={ }_{-.08}^{+.11}$, and $\sigma_{\mathrm{OMB}}={ }_{-.05}^{+.08}$.

The objects discovered by NEOWISE that have not had optical follow-up will add a significant bias to the PRAD as these objects are most likely to be ones missed by optical surveys, i.e., lower albedo asteroids. During the cryogenic portion of the survey, NEOWISE observed 23,616 previously unknown MBAs with data of sufficient quality for thermal modeling and with orbital arcs longer than one day (and thus not considered "one-night stand" observations by the MPC). Some $\sim 10,000$ additional asteroids were given temporary designations by the MPC but did not have sufficient arc lengths to calculate an orbit. While they do not have known orbits and thus cannot be classified as members of the Main Belt or not, we can use this to set an upper limit on the number of lost MBAs. As these objects are linked to older precovery data or are serendipitously followed up our count of discovered objects with computed orbits will increase. Out of these discovered objects 19,178 have optical photometric data as well as thermal infrared, allowing for albedo determination. 


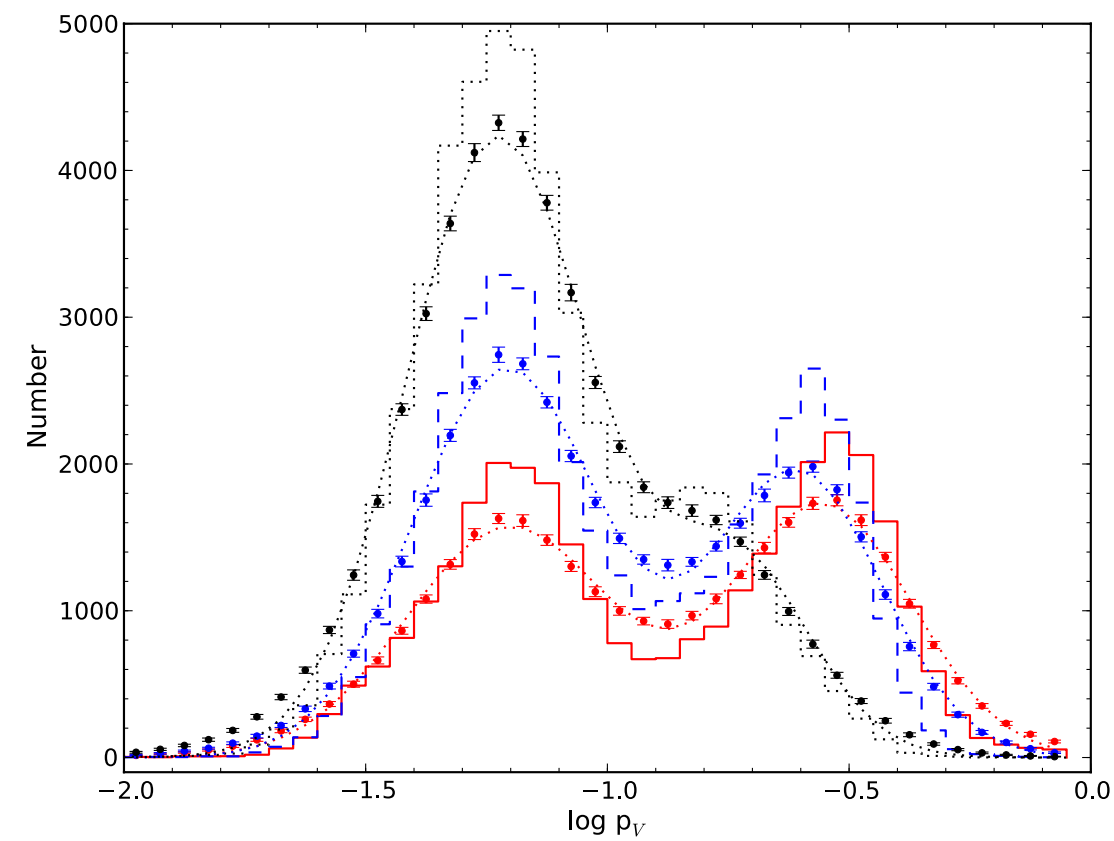

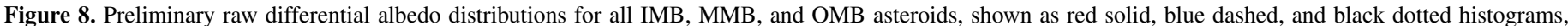

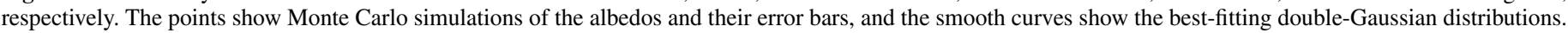
(A color version of this figure is available in the online journal.)

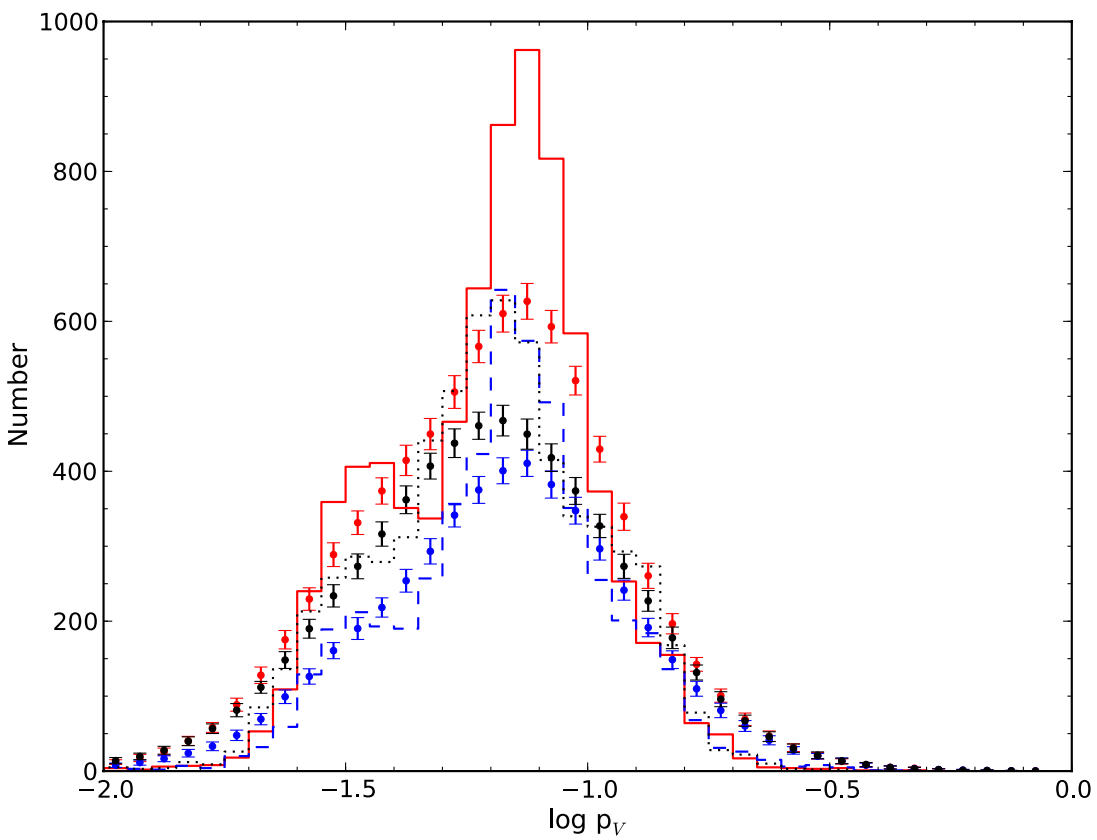

Figure 9. Same as Figure 8, but only for the NEOWISE-discovered MBAs that also received optical photometric follow-up.

(A color version of this figure is available in the online journal.)

It should be noted that while many of the discovered objects have optical observations, there is a strong bias in favor of recovery of the highest albedo discoveries by subsequent ground-based observations. The visible light received from an object is directly proportional to the albedo of that object, while the thermal infrared flux is driven by the temperature of the surface, which is only weakly dependent on albedo as shown in Equation (1). As such, optical surveys show a significant albedo bias toward brighter objects for both discovery and recovery observations, while the NEOWISE infrared survey is more sensitive to detection of low albedo objects. Any survey will have inherent biases in the data set and it is necessary to account for them before the true albedo distribution can be determined for a population.

We show in Figure 9 the albedo distribution of the NEOWISEdiscovered MBAs with optical photometry for the IMB, MMB, and $\mathrm{OMB}$ subpopulations. Even though the optical followup will be biased toward favoring higher albedo objects, the NEOWISE discoveries are dominated by low albedos, as these are the objects that were initially missed by the ground-based 


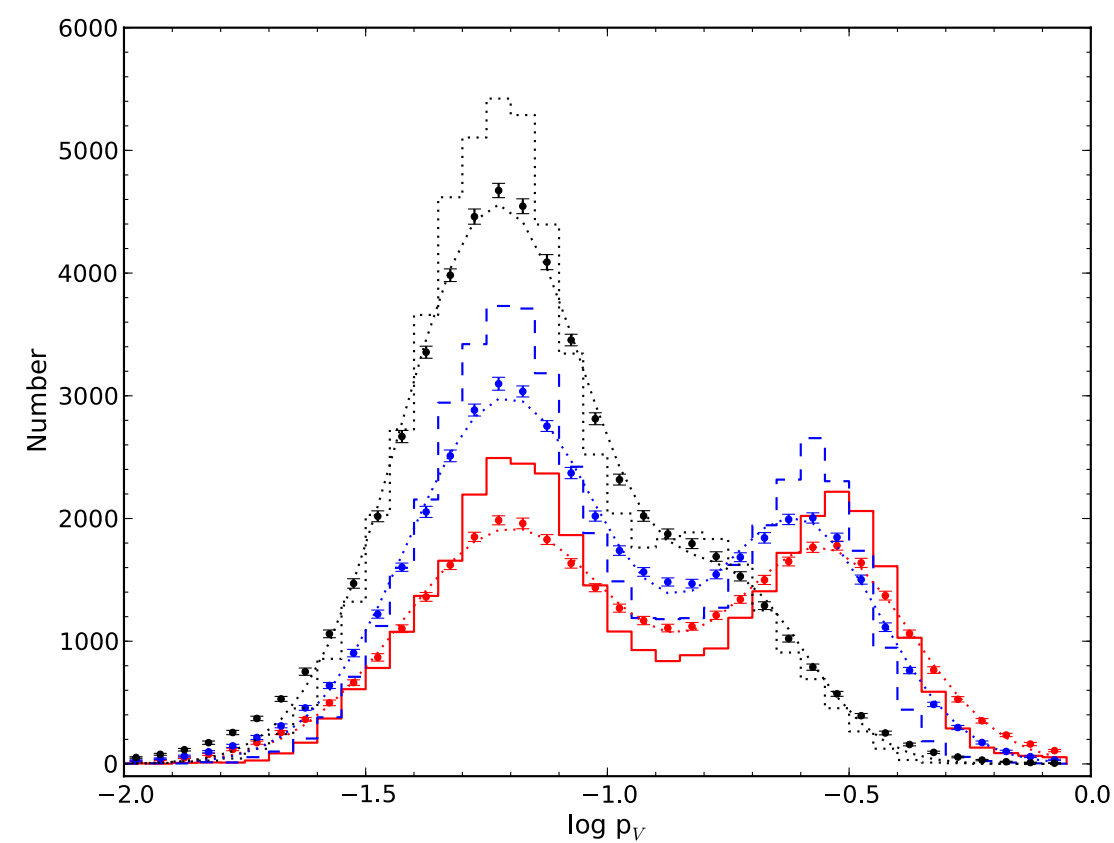

Figure 10. Same as Figure 8, but now including a simple model for the objects discovered by NEOWISE that have no optical follow-up photometry.

(A color version of this figure is available in the online journal.)

optical surveys. All three distributions can be described by a single Gaussian function with mean albedo between $0.05<$ $\mu<0.06$ and $0.02<\sigma<0.03$, consistent with the values found for the dark complex in the whole population above. We can use the albedos of the discovered objects and the roughly equal number of objects in each subgroup as an initial attempt to constrain the effect of these lost objects on the greater albedo distribution. We show in Figure 10 a revised albedo distribution including this toy model for the albedos of the $\sim 15,000 \mathrm{MBAs}$ without follow-up photometry, based on the albedo distribution of the NEOWISE-discovered objects. As expected the primary effect is to increase the relative abundance of dark objects in each region of the Belt.

We note that the more detailed way to properly account for the uncertainties introduced by objects without follow-up and objects for which reliable orbits could not be determined is through careful modeling of survey biases. A debiased study of the MBAs will be the subject of future work, and will allow us to determine the true size and albedo distributions of these objects.

\section{DYNAMICALLY GROUPED ALBEDOS IN THE MAIN BELT}

We also investigate the distribution of albedo as a function of orbital parameters, in particular semimajor axis $(a)$, eccentricity $(e)$, and inclination $(i)$. Figure 11 shows the distribution of albedos as a function of semimajor axis. We have color-coded the points by albedo using a "weather-map" palette divided evenly in $\log \left(p_{V}\right)$ space, and use this same color code for all subsequent plots. Dark colors (black, gray, dark blue, blue) indicate objects in the low albedo complex, while brighter colors (magenta, red, orange, yellow) indicate members of the high albedo complex. Objects colored yellow have very high albedos and are concentrated in the Hungaria region and IMB near the orbit of (4) Vesta. These largest albedos found are likely artifacts of using $G=0.15$ to calculate the $H$ value: while the thermal models for the diameters of these objects show no errors, using the literature $H$ value forces anomalously high albedos. We investigate the use of different $H$ and $G$ values for these highest albedo objects in a future publication.

We show in Figures 12 and 13 the plot of semimajor axis versus inclination and eccentricity, respectively, using the same colors denoted above. Asteroid albedos are not homogeneously distributed throughout the Main Belt, but rather are clumped in $a-e-i$ space, correlating with the positions of known asteroid families (Nesvorný et al. 2006a). MBAs have been previously shown to cluster in color space (Ivezić et al. 2002; Parker et al. 2008) and show similar reflectance spectra (e.g., Binzel \& Xu 1993; Cellino et al. 2001, etc.) indicative of the common origin of members of families, which are the result of a catastrophic breakup of a single parent body (Hirayama 1918). The clustering of albedos is a further confirmation of this origin. A future paper in this series will investigate the use of albedo as an added criterion to the orbital parameters typically used to determine family membership.

\section{IR REFLECTANCE}

The reflectance spectrum for most asteroids (but not all, e.g., B-types) show an increasing value as the wavelength moves from the visible to the NIR region of the spectrum (e.g., DeMeo et al. 2009, etc.). If these trends continue into the $W 1$ and $W 2$ bandpasses we would expect the reflectance observed there to be higher than observed in the optical. We assume in our thermal model that the reflectance in $W 1$ and $W 2$ is the same; depending on the location and depth of absorption bands this may not be universally true, but this assumption provides a generic constraint from which we can identify interesting objects that do not follow this assumption. Reflectance depends on both the albedo and the $G$ slope parameter, both of which cannot be assumed to be wavelength independent. We do not have sufficient phase coverage to fit $G_{\text {NIR }}$ and thus disentangle its effect from that of $p_{\mathrm{NIR}}$, so we present only the NIR reflectance ratio for objects in the Main Belt with sufficient signal in $W 1$ and/or $W 2$ to be able to fit this value, for a total of 4194 objects. 


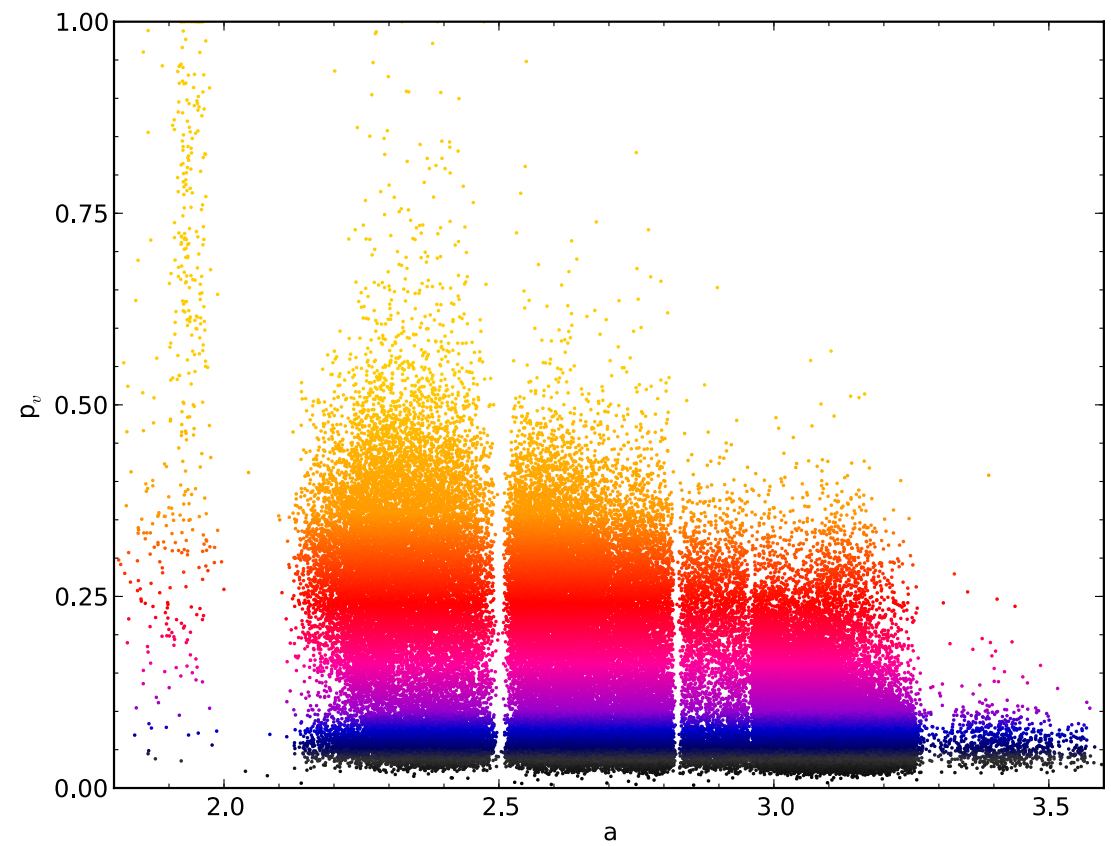

Figure 11. Asteroid albedo vs. semimajor axis. Colors denoted here are split evenly in $\log \left(p_{V}\right)$ space and are used to denote albedo in subsequent plots. (A color version of this figure is available in the online journal.)

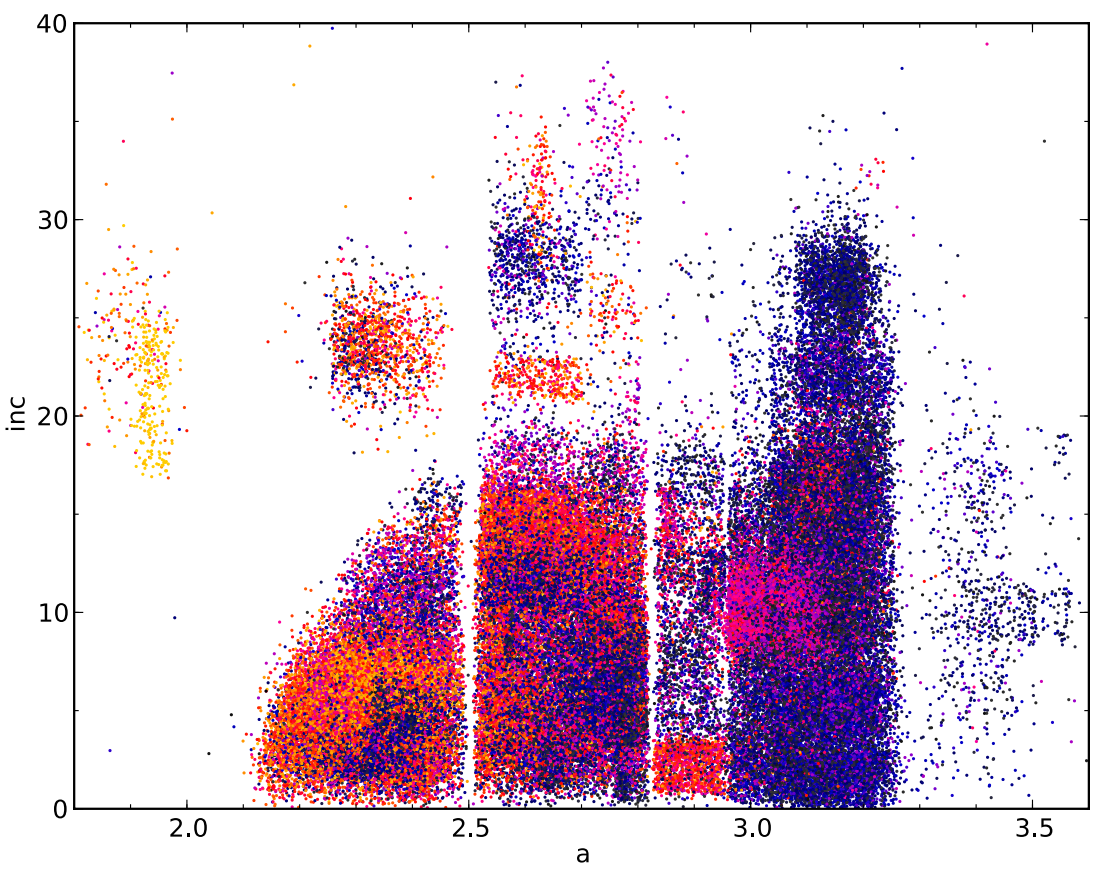

Figure 12. Orbital inclination vs. semimajor axis. Colors are the same as Figure 11.

(A color version of this figure is available in the online journal.)

We show in Figure 14 plots of the NIR reflectance ratio compared to a range of physical and orbital parameters, as well as running averages for those distributions. All objects were detected in thermal emission; however, only objects with sufficient reflected light were able to provide fitted NIR reflectance ratios. As such objects with higher IR albedos will be more likely to have a fitted reflectance ratio (this is comparable to the biases inherent in optical surveys). Evidence of this is apparent in Figure 14(e), where the running average of the ratio climbs for objects with fainter $H$ values. We note that as the visible albedo $p_{V}$ is intricately linked with the NIR/Vis ratio it cannot be considered an independent variable. The structure seen in the running average in Figure 14(f) is expected to be heavily influenced by the debiasing of the Main Belt population currently being undertaken. Future work (Mainzer et al. 2011c) will explore the connection between taxonomic types derived from spectroscopy and photometry and the IR reflectance ratio found in the WISE data.

In Figure 15 we show the raw differential distribution of NIR reflectance ratios for the IMB, MMB, and OMB asteroids with fitted ratios. Also shown are the mean distribution and associated errors derived from a one-hundred-trial MC simulation of all 


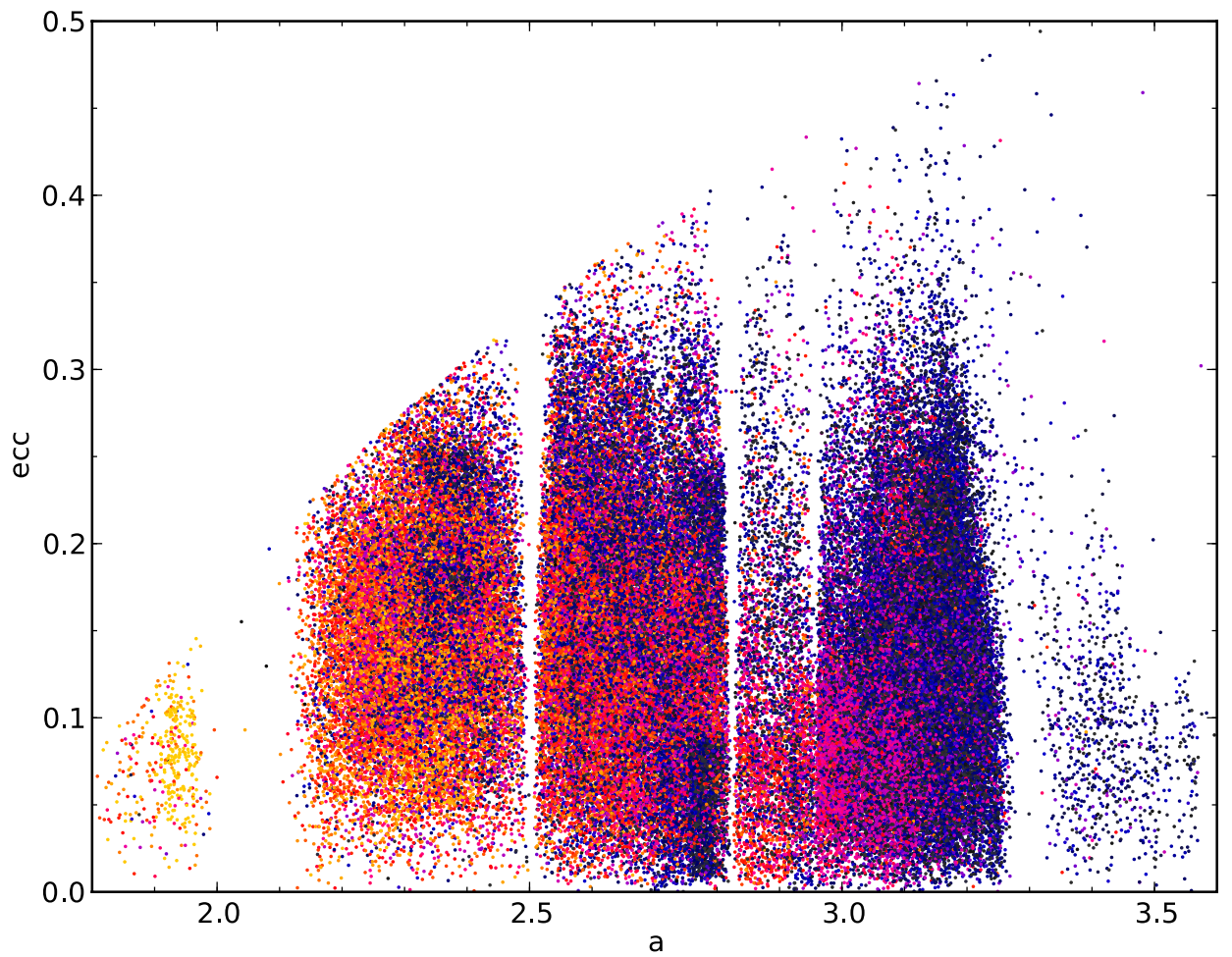

Figure 13. Orbital eccentricity vs. semimajor axis. Colors are the same as Figure 11.

(A color version of this figure is available in the online journal.)
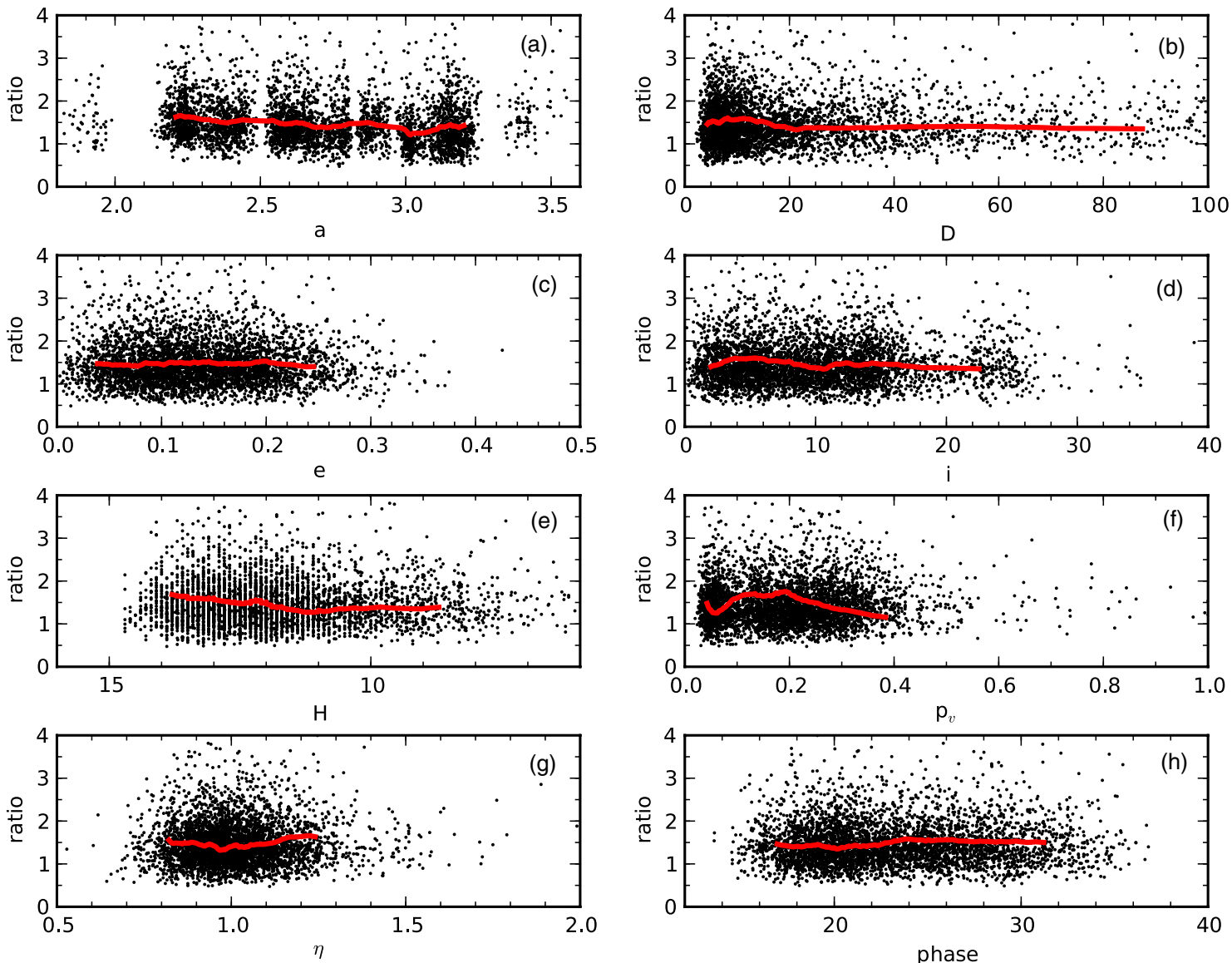

Figure 14. NIR reflectance ratio for all objects with fitted values, compared to (a) semimajor axis, (b) diameter, (c) eccentricity, (d) inclination, (e) absolute magnitude, (f) albedo, (g) beaming parameter, and (h) phase. The thick red line shows the running average for 400 object-wide bins stepped by 40 objects. The picket-fence effect in the absolute magnitude is an artifact of the 0.1 mag accuracy of $H$ for most objects.

(A color version of this figure is available in the online journal.) 


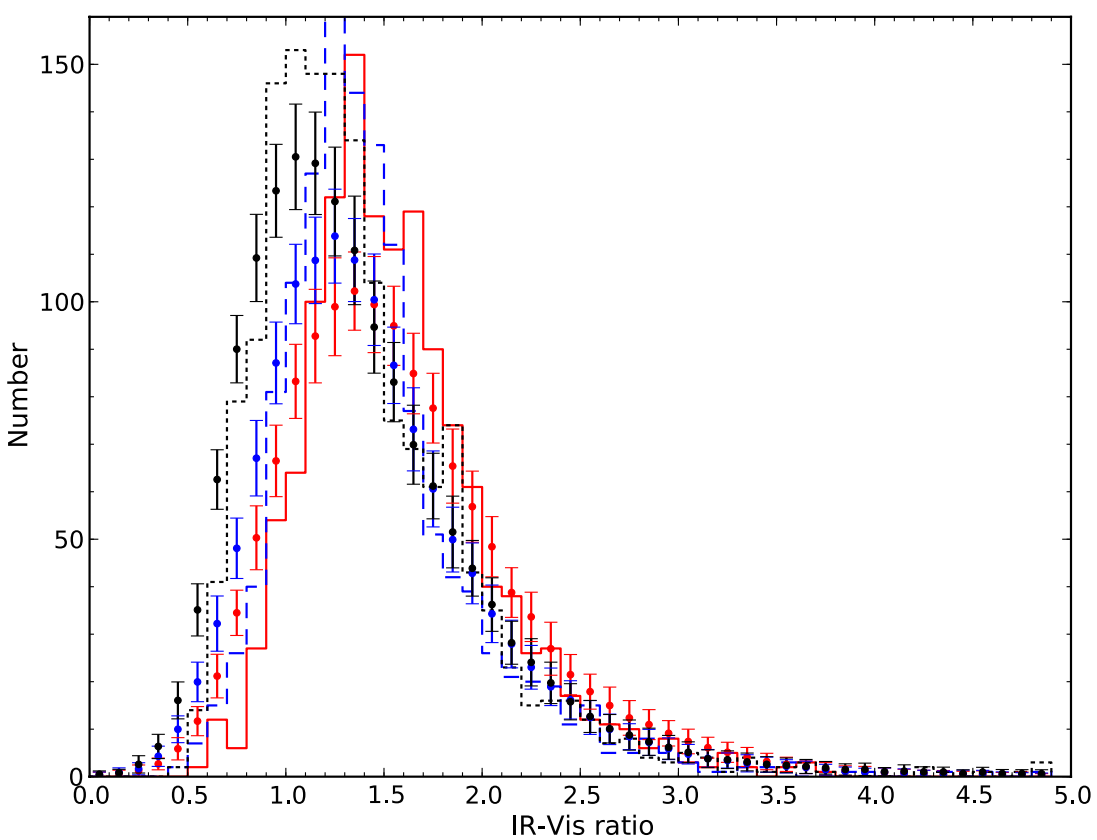

Figure 15. Distribution of NIR reflectance ratio for the IMB (red solid), MMB (blue dashed), and OMB (black dotted) populations. Shown as points are the mean distribution and associated errors from Monte Carlo simulations of each ratio.

(A color version of this figure is available in the online journal.)

measured reflectance ratios. All three populations show a peak between $1.2<$ ratio $<1.4$ while the running average versus semimajor axis varies across the Main Belts from $1.3<$ ratio $<$ 1.6. This is consistent with the values found for the NEOs by A. K. Mainzer et al. (2011, in preparation). For objects without fitted NIR reflectance ratios, we use the mean of all fitted values of 1.5 and an error bar based on the associated standard deviation of $\sigma_{\text {ratio }}=0.5$ for modeling purposes.

\section{ASTEROID FAMILIES}

Asteroid families were first identified as groups of objects that clustered tightly in orbital element-space by Hirayama (1918) nearly a century ago. Subsequent work has confirmed that families originate from the catastrophic breakup of a single parent asteroid after an impact (see Cellino et al. 2009 for a recent review of the current state of the field). This single mineralogical origin causes families to cluster tightly not only when comparing orbital elements but also when investigating colors (Ivezić et al. 2002; Parker et al. 2008) and reflectance spectra (e.g., Binzel \& Xu 1993; Cellino et al. 2001, etc.). The SFD of asteroid family members can also act as a tracer of the physical properties of the original parent body and can even be used to constrain the impact velocity and angle (Durda et al. 2007). However, a major deficiency in models to date has been the lack of measured diameters for the family members, forcing these values to be assumed based on the apparent magnitude of the object. Albedo measurements of the largest bodies in a family are often available from the IRAS data set (Tedesco et al. 2002) and can be used to assume an albedo for all family members, but this can add a significant and systematic error to the diameters used, especially in the cases where families may be mixed or where the largest body in a family may not be associated with the other members (e.g., Cellino et al. 2001).

There are a number of methods that can be used to determine which asteroids are members of a given family. The Hierarchical Clustering Method (HCM; Zappalà et al. 1990), a commonly used technique, takes the differences in velocities between the proper orbital elements of objects to reveal dynamical associations. Nesvorný (2010) used this method to identify 55 families out of 293,368 MBAs with low inclinations. We use these 55 families as the baseline for our analysis, selecting those objects that appear both in that list and in the WISE observations. As all these objects were discovered by optical surveys, there will be an inherent bias in the albedos favoring brighter family members. Future work will address this bias and explore the use of albedo in conjunction with dynamical orbital properties to identify members of asteroid families and to reject interloper objects.

Of the 55 families identified by Nesvorný (2010) we find that 46 have more than 20 members observed during the cryogenic WISE mission. Due to the limitations of proper orbital element calculations, high inclination objects are not included in the AstDys list ${ }^{19}$ of proper orbital elements (Milani \& Kneżević 1994). As such, high inclination families are likewise not represented in the list of family members. We have, however, included in our analysis the Pallas family, identified by D. Nesvorny (2011, private communication) through the same methods as the 55 published families. We also include the asteroids located in the Hungaria region; while not canonically included in the list of dynamical families, recent work by Warner et al. (2009b) and Milani et al. (2010) support the classification of objects near Hungaria in orbital space as a true dynamical family. These two added groups bring our total considered population up to 48 families. We show in Figure 16 the proper inclination against the proper semimajor axis for all objects identified as a member of one of the families considered here. Note that for the Hungaria and Pallas families proper orbital elements were not available and so osculating elements were used. Similarly, we show in Figure 17 the proper eccentricity against the proper semimajor axis. In both plots, we use the same color scheme as shown in Figure 11. It is quite apparent in

\footnotetext{
19 http://hamilton.dm.unipi.it/astdys/index.php
} 


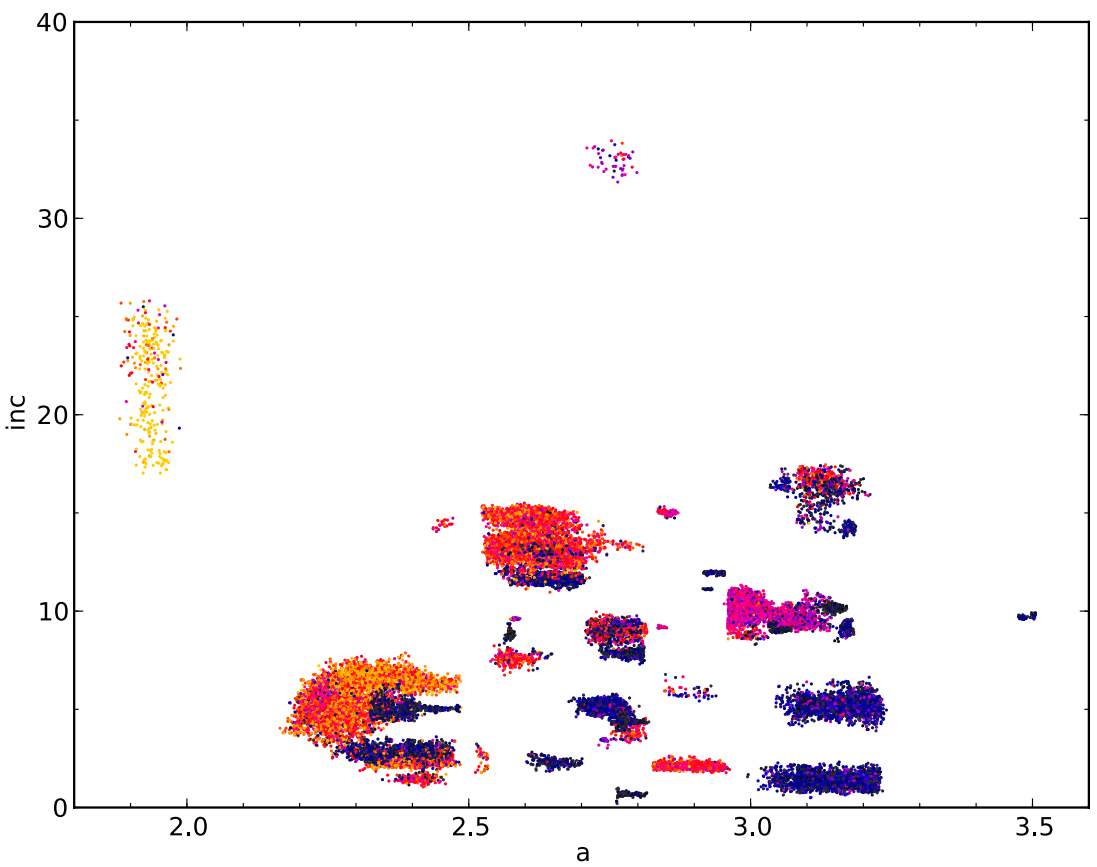

Figure 16. Proper orbital inclination vs. proper semimajor axis for asteroid families. Colors are the same as Figure 11.

(A color version of this figure is available in the online journal.)

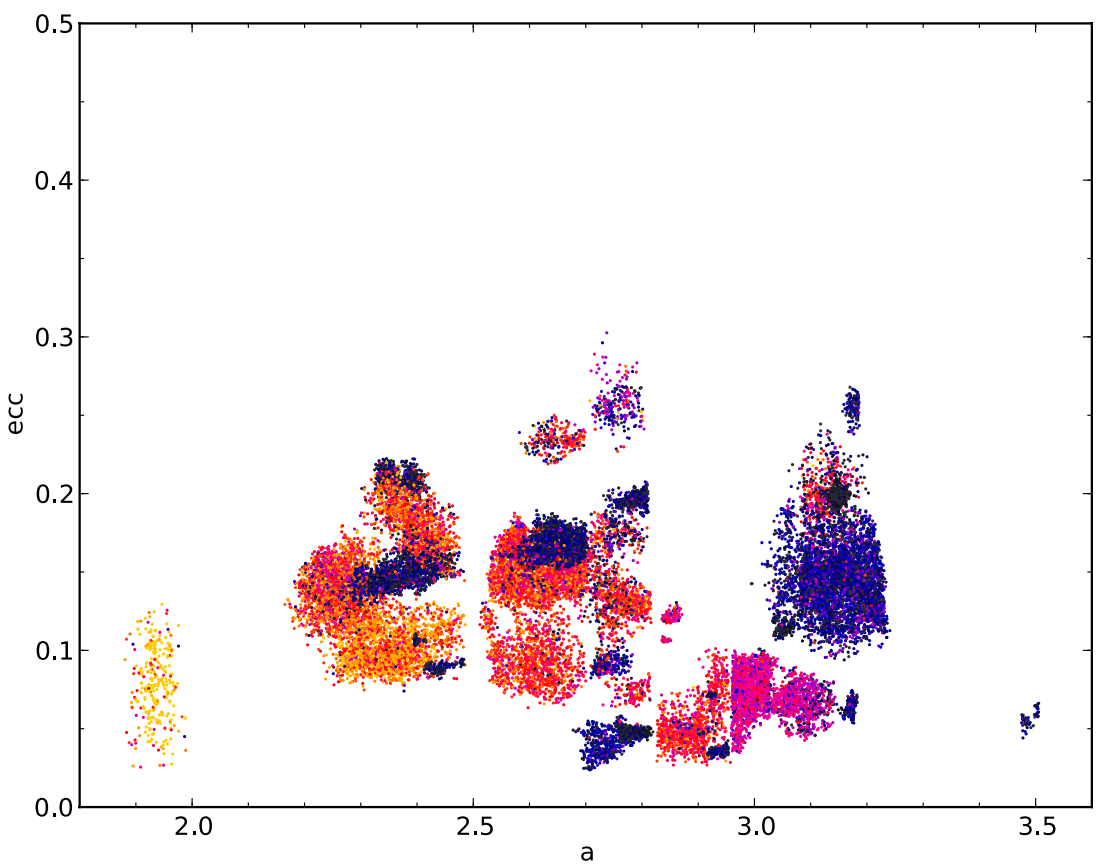

Figure 17. Proper orbital eccentricity vs. proper semimajor axis for asteroid families. Colors are the same as Figure 11.

(A color version of this figure is available in the online journal.)

these plots that families characteristically have uniform albedos; however, there are notable exceptions.

We show in Figure 18 the cumulative PRSFD for each of the families observed during the WISE survey, as well as MC simulations of all distributions with appropriate error bars. Without debiasing, the PRSFD cannot be assumed to represent the true size distribution of the entire family population, as neither the NEOWISE-inherent biases nor the biases in family selection have been accounted for. Approximately $25 \%$ of these families show kinks at the large end of the distribution inconsistent with a simple power law. As small number statistics dominate families especially at the largest sizes, and because WISE did not survey the entire Main Belt before the exhaustion of cryogen, precise debiasing is required to confidently measure the shape of the true SFD, especially at the largest sizes for each family.

In Figure 19 we show the normalized PRAD for each family, along with the MC results for each distribution. As was evident in Figure 16 and Figure 17 most families show a single-peaked albedo distribution; however, as mentioned above, debiasing will be critical to proper interpretation of these distributions. About $15 \%$ of families include a small 


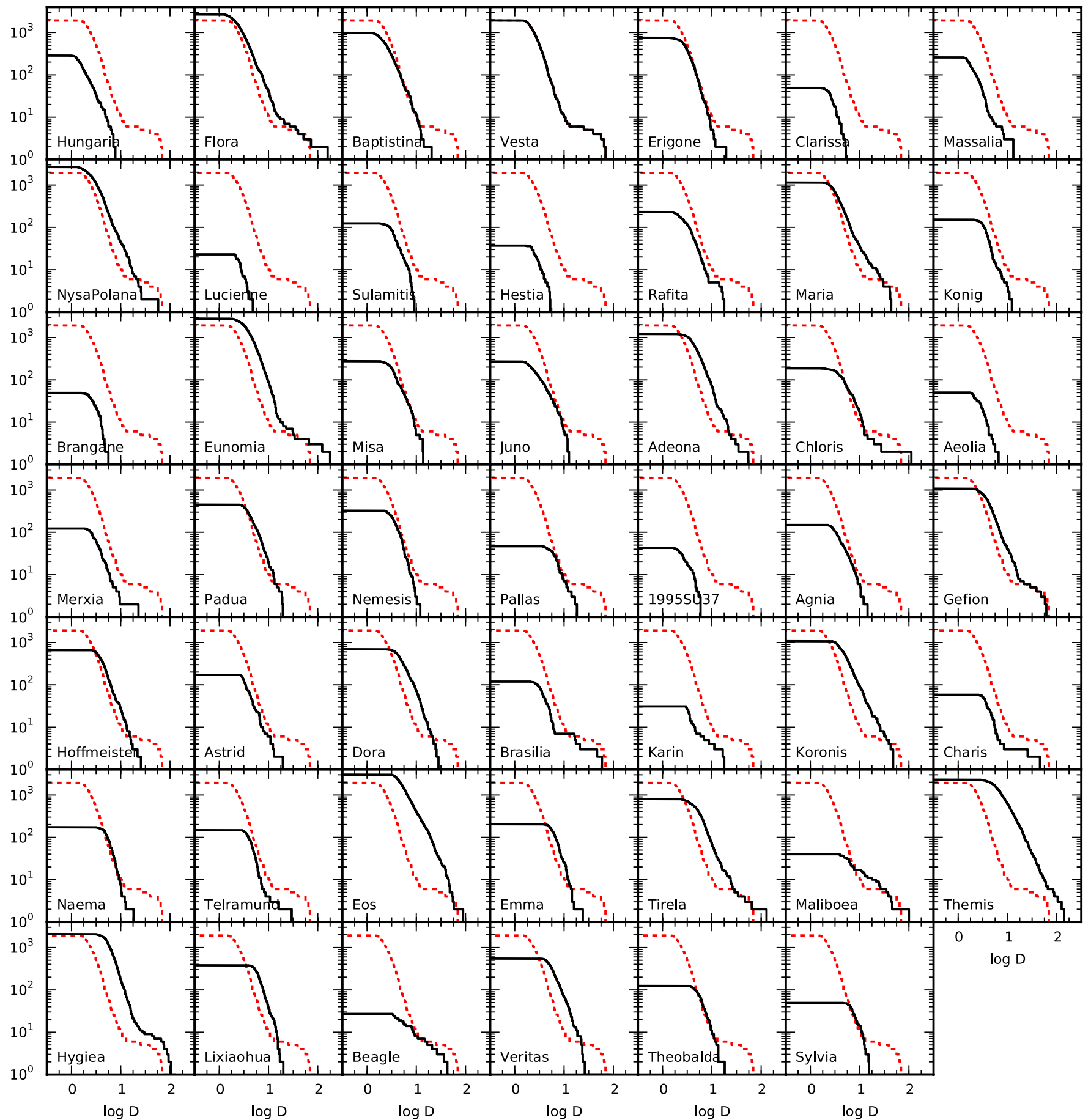

Figure 18. Cumulative raw size-frequency distribution for each asteroid family considered here. The solid black line shows the family indicated by the name in each plot. The dotted red line shows the PRSFD for the Vesta family in all plots, for ease of comparison.

(A color version of this figure is available in the online journal.)

population of objects with non-characteristic albedos; these may be the result of an improper association of background objects into the family. However, another $10 \%$ of families show significant mixing between two albedo types that cannot be solely due to the intrusion of a few background objects. In particular, the Nysa-Polana and Tirela families show near-parity between the low- and high-albedo objects in the preliminary raw distributions.

Among the families with sufficient data for study were the Karin and Koronis families. The Karin family is believed to be a very young family, with an age of $\sim 5.8 \mathrm{Myr}$, that formed from the breakup of a member of the much older (2-3 Gyr) Koronis family (Nesvorný et al. 2006b; Harris et al. 2009). We fit a Gaussian to the observed family albedo distributions. We find that the Karin family has a lower mean albedo from this fit ( $\left.p_{V-\text { Karin }}=0.18 \pm 0.05\right)$ than the Koronis family $\left(p_{V-\text { Koronis }}=0.24 \pm 0.05\right)$ where the error bars represent the width of the best-fit Gaussian. Chapman (2004) gives an overview of space weathering effects, a process that is generally thought to darken and redden surfaces of atmosphereless bodies 


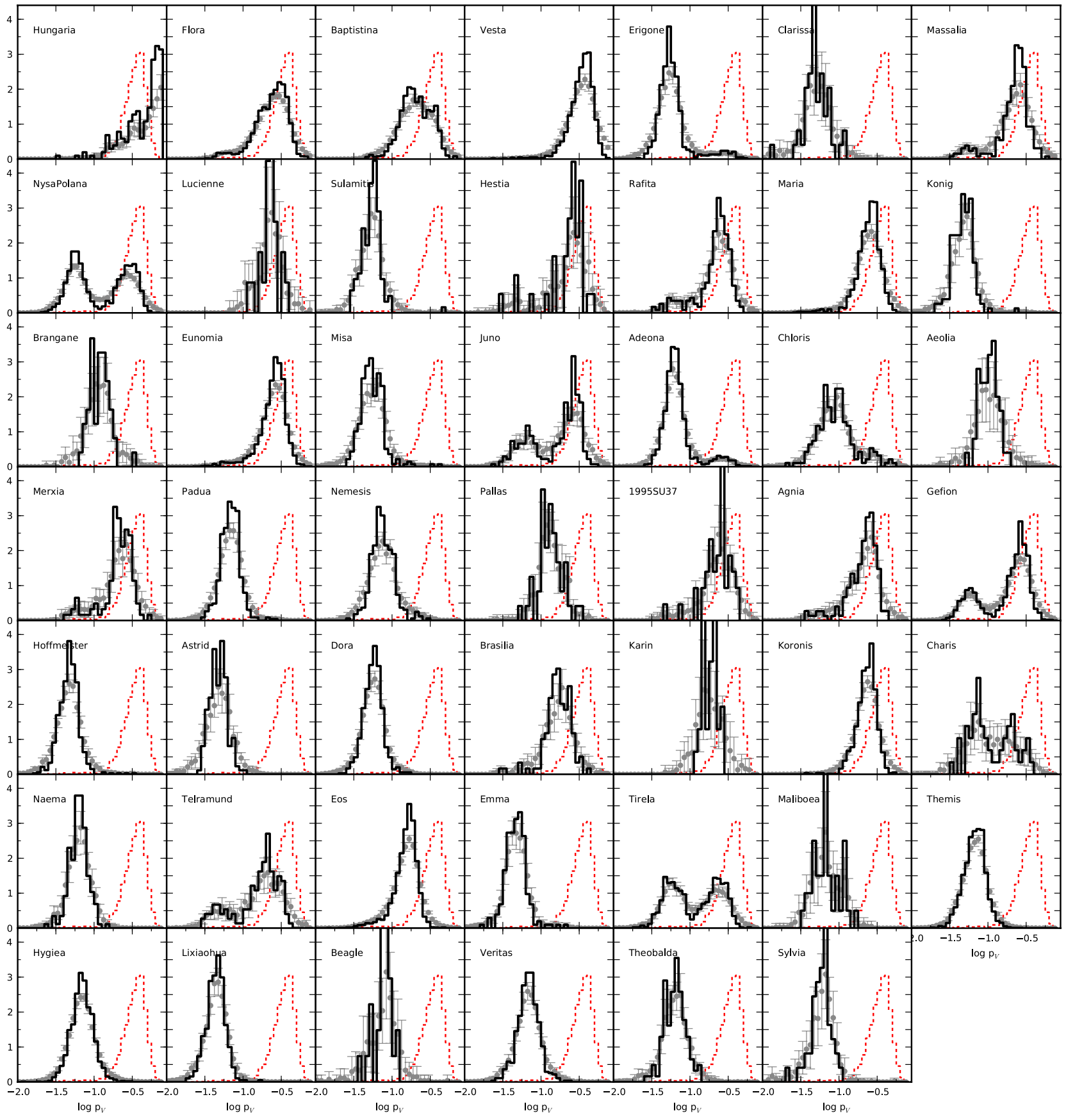

Figure 19. Same as Figure 18 but for the PRAD. Monte Carlo simulations of the distributions are plotted as gray points with error bars. The distributions have been normalized to unit area for easier comparison.

(A color version of this figure is available in the online journal.)

in the solar system. Our result is in apparent contradiction with this hypothesis under the assumption that the compositions of both families are identical. It is possible that variations in the composition, the presence of interlopers, or differentiation of the proto-Koronis parent body could result in this observation. This analysis will be strengthened by the identification of more NEOWISE-observed family members (there were 31 identified members of the Karin family and 1079 members of the Koronis family in this data set) and the acquisition of additional compositional information.

We also observed 984 asteroids that were identified as members of the Baptistina family. Bottke et al. (2007) postulate that a fragment from the breakup of the Baptistina family was the impactor responsible for the $K / T$ mass extinction event. However, these authors assumed an albedo for the family members of 0.05 . We find that the best-fitting Gaussian to the Baptistina family member albedos has a mean of $p_{V-\text { Baptistina }}=$ $0.21_{-0.08}^{+0.13}$ where the error bar indicates the width of the Gaussian distribution. The method of age determination used by Bottke et al. (2007) depends on the albedo assumed, and the calculated age $T$ is proportional to albedo following $T \propto p_{V}^{-0.5}$. Thus our measured albedo results in an age for the breakup approximately $\sim 2$ times younger than that found with the lower assumed albedo (from $160 \mathrm{Myr}$ to $\sim 80 \mathrm{Myr}$ ), reducing the likelihood that the Baptistina breakup generated the $\mathrm{K} / \mathrm{T}$ impactor (cf. Reddy et al. 2009; Carvano \& Lazzaro 2010). 
Also of interest are the families that show characteristic albedos distinct from the distribution observed in the Main Belt in Figure 8. For example, the Eos and Aeolia families have characteristic albedos that fall in between the two peaks of the MBA albedos. Standing out as an outlier is the Hungaria family: while showing a fairly strong coherence in albedo within the family, that characteristic albedo is incredibly high (see below). Future work will investigate whether variations in the $G$ slope parameter used to compute the $H$ absolute magnitude (and thus the reflected-light albedo) from the assumed value of $G=0.15$ typically used could account for the very large albedos calculated in these preliminary results.

\section{UNUSUAL OBJECTS}

A small fraction of our objects had fits that did not conform to the general trends seen for the population as a whole. These highly unusual fits may be caused by strange physical parameters, incorrect associated properties (e.g., $H$ mag, orbital elements, etc), or a breakdown of the NEATM model. In any case, these objects warrant further inspection.

The most obvious candidates for this category of unusual fits are the asteroids with very high visible geometric albedos $\left(p_{V} \geqslant 0.70\right)$. We find 193 objects in our survey have computed albedos that fall into this range, mostly contained within the region of orbital element space occupied by the Hungaria and Vesta families (out of 343 objects found in the Hungaria region and 1938 in the Vesta family), implying a possible mineralogical origin.

The asteroid (434) Hungaria, the lowest numbered member of its namesake region, has a relatively large albedo of $p_{V}=0.46$, which can be explained by a composition dominated by the ironpoor mineral enstatite; however, some of the observed spectral features may require contamination from a darker, external source (Kelley \& Gaffey 2002). This may indicate that albedos can range larger than $p_{V} \sim 0.5$, but albedos significantly higher than this are likely suspect.

Visual inspection of the thermal model fits of these objects shows that these high albedos are not due to a failure of the thermal model. For those objects where IR reflectance factor could also be fitted, we find values typically with reflectance ratio $\leqslant 1$, while the distribution of beaming parameters for these objects is similar to that of the general Main Belt population. If the $H$ or $G$ values for these objects were very far from the true values this could result in the unusual calculated albedos.

By increasing the error bar we assume for the $H$ value and setting the IR reflectance ratio to a constant value of ratio $=1.5$ (the average for MBAs) we are able to find good fits of objects with reflected light in $W 1$ and/or $W 2$. Under this assumption, these objects all return fits with albedos comparable to that of (434) Hungaria. However, all these cases require the $H$ magnitudes to be $0.4-1.0$ mag fainter than the values given by the MPC.

A misidentification of $H$ could be a symptom of an improper assumption for the $G$ slope parameter. In particular, if $G$ should be much larger (e.g., equal to or greater than the value for (44) Nysa of $G=0.46^{20}$ ) this would result in an $H$ value that was too bright by $\sim 0.4$ mag or more, which would thus give an albedo that was a factor of $\sim 1.4$ too large. This can account for some, but not all, of our improbably high albedos. Large light-curve amplitudes may also contribute to an absolute magnitude that is too bright. Future work will address these objects in detail.

\footnotetext{
20 As given by the Small Body Database: http://ssd.jpl.nasa.gov/sbdb.cgi.
}

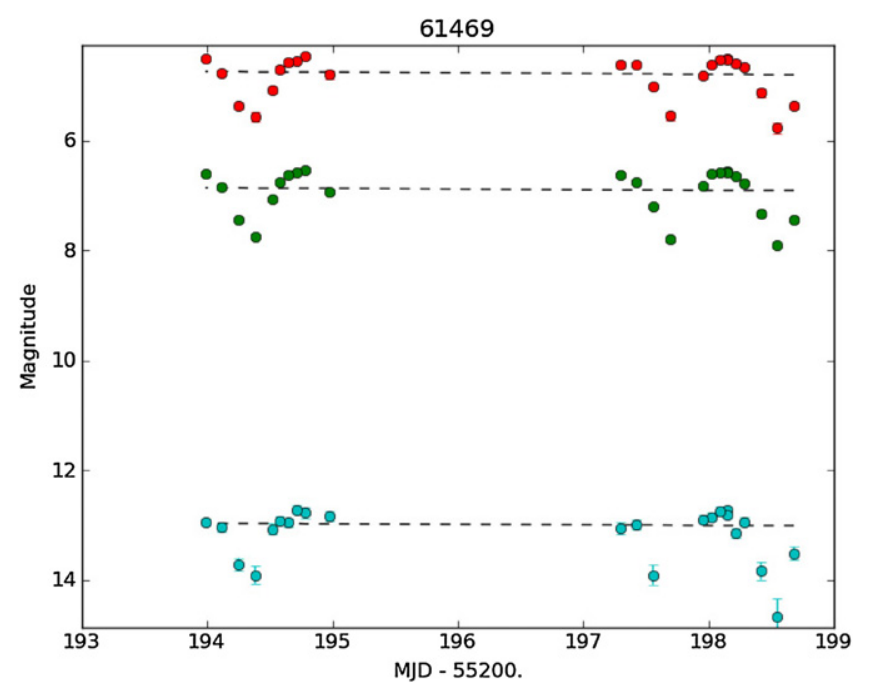

Figure 20. Light curve for asteroid (61469), with magnitudes for $W 4, W 3$, and $W 2$ shown in red, green, and cyan (top to bottom), respectively. The dashed line shows the modeled magnitudes for the best-fitting sphere. The period of this asteroid is approximately $\sim 40 \mathrm{hr}$ assuming a double-peaked light curve.

(A color version of this figure is available in the online journal.)

In addition to the high albedo objects, we find a very small number of objects with beaming parameters at or close to the theoretical limit of $\eta=\pi$. While some of these fits can be rejected upon visual inspection, e.g., because of a single bad point in $W 4$ dragging the fit to higher beaming parameter, at least six objects appear to be legitimate fits with beaming parameters indicative of latitudinally isothermal surfaces, though none of the fits show any significant light-curve variations. Further investigation will be critical to determine if they show rapid rotation or very high thermal inertia needed to explain this beaming parameter.

Approximately 10,000 MBAs were observed by WISE at two different epochs. We initially treated each epoch separately for fitting purposes, and in the majority of cases the fits were within the expected error of each other. For those that were not, we recomputed the best-fitting model using both epochs together and forcing the physical parameters to be identical. We find 36 objects for which the two-epoch fits could not produce a good fit at one or both epochs. While some of these objects may have very long rotation periods $(P>10$ days $)$ and thus different projected diameters between epochs, others do not appear to show any light-curve variation during our observations and may be cases of objects showing a significant difference between the temperatures of the morning and afternoon hemispheres of the body.

We also observe 151 objects with peak-to-trough light-curve variations larger than $1.5 \mathrm{mag}$ in $W 3$ and average magnitude measurement errors smaller than $\sigma_{W 3}<0.2$ after removing spurious measurements. As these are not fitted amplitudes they represent a minimum for the light-curve amplitude for the body observed. An example of one of these objects, (61469), is shown in Figure 20. There are also many objects with amplitudes smaller than this cutoff with readily apparent rotational effects, too. A future work will investigate specific light curves to determine the period and amplitude of the objects.

\section{CONCLUSIONS}

We have presented an initial analysis of MBAs detected by NEOWISE during the cryogenic portion of the WISE mission. 
With infrared fluxes of sufficient quality to determine diameters and albedos for 129,750 MBAs, we show the power and great potential contained in this data set. These data allow us to probe the composition, structure, and history of the Main Belt in ways that were previously impossible.

For objects with thermal emission detected in two or more bands we allowed the beaming parameter to vary. We find a mean beaming parameter of $\eta \sim 1.0$; however, we do see evidence of a phase dependence for the beaming parameter, ranging from $\eta \sim 0.94$ for low phases to $\eta \sim 1.14$ for higher phases (within the Main Belt). The best-fit linear relation between beaming and phase is $\eta=0.79+0.011 \alpha$, which is a much shallower relation than seen previously in Wolters \& Green (2009), but consistent with A. K. Mainzer et al. (2011, in preparation) which includes the NEOs with fitted beaming parameters as well.

As was observed in the IRAS data (e.g., Tedesco et al. 1989, 2002, 2005), the albedos of MBAs are strongly bimodal: a bright complex $\left(p_{V} \sim 0.25\right)$ and a dark complex $\left(p_{V} \sim 0.06\right)$. We find both peaks to be described well by Gaussian distributions in log-albedo space.

We find that the reflectance of asteroids in the $W 1$ and $W 2$ bandpasses is typically larger than the albedo found in visible light. The best-fit ratio of reflectance ranges from $\sim 1.6$ in the IMB to $\sim 1.3$ in the OMB; however, the spread of values is large, and the final distribution will depend strongly on the debiasing.

We identify albedo clusters in $a-e-i$ space corresponding to the locations of asteroid families. Albedo is another coherent property of dynamical families in addition to orbit (Hirayama 1918), color (Ivezić et al. 2002), and reflectance spectrum (e.g., Binzel \& Xu 1993; Cellino et al. 2001, etc.). Albedo can also be used to trace the halos of similar objects that surround some families (e.g., Vesta, Eos, etc.; Parker et al. 2008) that may be evidence of a collisional breakup very early in the age of the solar system. Using asteroids previously identified though HCM techniques to be members of collisional families, we show that most, but not all, families have a characteristic albedo.

Critical to any interpretation of the observations presented here is a careful accounting of the biases in both the WISE survey data as well as the optical data used to derive albedos. We are currently undertaking an extensive debiasing campaign with the goal of producing unbiased size and albedo distributions for the Main Belt. This will be the subject of the next paper in this series.

We thank the anonymous referee for the helpful comments and suggestions that led to the improvement of this manuscript. J.R.M. was supported by an appointment to the NASA Postdoctoral Program at JPL, administered by Oak Ridge Associated Universities through a contract with NASA. J.R.M. thanks M. Delbó and M. Mueller for providing access to their thermal modeling code which was helpful in early test cases. This publication makes use of data products from the Wide-field Infrared Survey Explorer, which is a joint project of the University of California, Los Angeles, and the Jet Propulsion Laboratory/ California Institute of Technology, funded by the National Aeronautics and Space Administration. This publication also makes use of data products from NEOWISE, which is a project of the Jet Propulsion Laboratory/California Institute of Technology, funded by the Planetary Science Division of the National Aeronautics and Space Administration. This research has made use of the NASA/IPAC Infrared Science Archive, which is operated by the Jet Propulsion Laboratory/California Institute of Technology, under contract with the National Aeronautics and
Space Administration. We thank the worldwide community of dedicated amateur and professional astronomers devoted to minor planet follow-up observations. We are deeply grateful for the outstanding contributions of all members of the WISE and NEOWISE teams.

\section{REFERENCES}

Binzel, R. P., \& Xu, S. 1993, Science, 260, 186

Bottke, W. F., Durda, D. D., Nesvorný, D., et al. 2005a, Icarus, 175, 111

Bottke, W. F., Durda, D. D., Nesvorný, D., et al. 2005b, Icarus, 179, 63

Bottke, W. F., Vokrouhlický, D., \& Nesvorný, D. 2007, Nature, 449, 48

Bottke, W. F., Vokrouhlický, D., Rubincam, D. P., \& Nesvorný, D. 2006, Annu. Rev. Earth Planet. Sci., 34, 157

Bowell, E., Hapke, B., Domingue, D., et al. 1989, Asteroids II (Tucson, AZ: Univ. Arizona Press), 524

Bus, S. J., \& Binzel, R. P. 2002, Icarus, 158, 146

Carvano, J. M., \& Lazzaro, D. 2010, MNRAS, 404, L31

Cellino, A., Del'Orro, A., \& Tedesco, E. F. 2009, Planet. Space Sci., 57, 173

Cellino, A., Gil Hutton, R., Tedesco, E. F., di Martino, M., \& Brunini, A. 1999, Icarus, 138, 129

Cellino, A., Zappalà, V., Doressoundiram, A., et al. 2001, Icarus, 152, 225

Chapman, C. R. 2004, Annu. Rev. Earth Planet. Sci., 32, 539

Chapman, C. R., Morrison, D., \& Zellner, B. 1975, Icarus, 25, 104

Cutri, R. M., Wright, E. L., Conrow, T., et al. 2011, http://wise2.ipac.caltech.edu/ docs/release/prelim/expsup/wise_prelrel_toc.html

DeMeo, F. E., Binzel, R. P., Slivan, S. M., \& Bus, S. J. 2009, Icarus, 202, 160

Durda, D. D., Bottke, W. F., Nesvorný, D., et al. 2007, Icarus, 186, 498

Gladman, B. J., David, D. R., Neese, C., et al. 2009, Icarus, 202, 104

Gradie, J., \& Tedesco, E. 1982, Science, 216, 1405

Grav, T., Mainzer, A. K., Bauer, J. M., et al. 2011, ApJ, in press

Harris, A. W. 1998, Icarus, 131, 291

Harris, A. W., \& Lagerros, J. S. V. 2002, Asteroids III, ed. W. F. Bottke, Jr., A. Cellino, P. Paolicchi, \& R. P. Binzel (Tucson, AZ: Univ. Arizona Press), 205 Harris, A. W., Mueller, M., Lisse, C. M., \& Cheng, A. F. 2009, Icarus, 199, 86 Hirayama, K. 1918, ApJ, 31, 185

Howett, C. J. A., Spencer, J. R., Pearl, J., \& Segura, M. 2010, Icarus, 206, 573

Ivezić, Ž., Lupton, R. H., Jurić, M., et al. 2002, AJ, 124, 2943

Ivezić, Ž., Tabachnik, S., Rafikov, R., et al. 2001, AJ, 122, 2749

Jedicke, R., \& Metcalfe, T. S. 1998, Icarus, 131, 245

Jurić, M., Ivezić, Ž., Lupton, R. H., et al. 2002, AJ, 124, 1776

Kelley, M. S., \& Gaffey, M. J. 2002, Meteorit. Planet. Sci., 37, 1815

Lebofsky, L. A., \& Spencer, J. R. 1989, Asteroids II (Tucson, AZ: Univ. Arizona Press), 128

Lebofsky, L. A., Sykes, M. V., Tedesco, E. F., et al. 1986, Icarus, 68, 239

Li, J.-Y., McFadden, L. A., Thomas, P. C., et al. 2010, Icarus, 208, 238

Liu, F., Cutri, R., Greanias, G., et al. 2008, Proc. SPIE, 7017E, 16

Mainzer, A. K., Bauer, J. M., Grav, T., et al. 2011a, ApJ, 731, 53

Mainzer, A. K., Eisenhardt, P., Wright, E. L., et al. 2006, Proc. SPIE, 6265, 61

Mainzer, A. K., Grav, T., Masiero, J., et al. 2011b, ApJ, 736, 100

Mainzer, A. K., Grav, T., Masiero, J., et al. 2011c, ApJ, in press

Milani, A., \& Knez̀ević, Z. 1994, Icarus, 107, 219

Milani, A., Kneżević, Z., Novaković, B., \& Cellino, A. 2010, Icarus, 207, 769

Morbidelli, A., Brasser, R., Gomes, R., Levison, H. F., \& Tsiganis, K. 2010, AJ, 140,1391

Morrison, D. 1974, ApJ, 194, 203

Mueller, T. G., Lellouch, E., Stansberry, J., et al. 2010, A\&A, 518, L146

Nesvorný, D. 2010, EAR-A-VARGBDET-5-NESVORNYFAM-V1.0, NASA

Planetary Data System, http://sbn.psi.edu/pds/resource/nesvornyfam.html

Nesvorný, D., \& Bottke, W. F. 2004, Icarus, 170, 324

Nesvorný, D., Bottke, W. F., Dones, L., \& Levison, H. F. 2002, Nature, 417, 720

Nesvorný, D., Bottke, W. F., Vokrouhlický, D., Morbidelli, A., \& Jedicke, R. 2006a, in IAU Symp. 229, Asteroids, Comets, Meteors, ed. L. Daniela, M. Sylvio Ferraz, \& F. J. Angel (Cambridge: Cambridge Univ. Press), 289

Nesvorný, D., Enke, B. L., Bottke, W. F., et al. 2006b, Icarus, 183, 296

O’Brien, D. P., \& Greenberg, R. 2003, Icarus, 164, 334

Ostro, S. J., Hudson, R. S., Benner, L. A. M., et al. 2002, Asteroids III, ed. W. F. Bottke, Jr., A. Cellino, P. Paolicchi, \& R. P. Binzel (Tucson, AZ: Univ. Arizona Press), 151

Parker, A., Ivezić, Ž., Jurić, M., et al. 2008, Icarus, 198, 138

Piazzi, G. 1801, Risultati delle Osservazioni della Nuova Stella Scoperta il di 1. Gennajo all'Osservatorio Reale di Palermo (Palermo: Nella Reale Stamperia) 
Reddy, V., Emery, J. P., Gaffey, M. J., et al. 2009, Meteorit. Planet. Sci., 44, 1917

Ryan, E. L., \& Woodward, C. E. 2010, AJ, 140, 933

Schmidt, B. E., Thomas, P. C., Bauer, J. M., et al. 2009, Science, 326, 275

Shevchenko, V. G., \& Tedesco, E. F. 2006, Icarus, 184, 211

Tedesco, E. F., Cellino, A., \& Zappalá, V. 2005, AJ, 129, 2869

Tedesco, E. F., Noah, P. V., Noah, M., \& Price, S. D. 2002, AJ, 123, 1056

Tedesco, E. F., Noah, P. V., Noah, M., \& Price, S. D. 2004, IRAS-A-FPA-3RDR-IMPS-V6.0, NASA Planetary Data System

Tedesco, E. F., Williams, J. G., Matson, D. L., et al. 1989, AJ, 97, 580
Walsh, K. J., \& Morbidelli, A. 2011, A\&A, 526, 126

Warner, B. D., Harris, A. W., \& Pravec, P. 2009a, Icarus, 202, 134

Warner, B. D., Harris, A. W., Vokrouhlický, D., Nesvorný, D., \& Bottke, W. F 2009b, Icarus, 204, 172

Wolters, S. D., \& Green, S. F. 2009, MNRAS, 400, 204

Wright, E. L. 2007, arXiv:astro-ph/0703085

Wright, E. L., Eisenhardt, P., Mainzer, A. K., et al. 2010, AJ, 140, 1868

Zappalà, V., Cellino, A., Farinella, P., \& Knezevic, Z. 1990, AJ, 100, 2030

Zellner, B. 1979, Asteroids (Tucson, AZ: Univ. Arizona Press), 783 